\title{
Option Pricing Under a Double Exponential Jump Diffusion Model*
}

\author{
S. G. Kou and Hui Wang \\ This version May 27, 2003
}

\begin{abstract}
Analytical tractability is one of the challenges faced by many alternative models that try to generalize the Black-Scholes option pricing model to incorporate more empirical features. The aim of this paper is to extend the analytical tractability of the BlackScholes model to alternative models with jumps. We demonstrate a double exponential jump diffusion model can lead to an analytic approximation for finite horizon American options (by extending the Barone-Adesi and Whaley method) and analytical solutions for popular path-dependent options (such as lookback, barrier, and perpetual American options). Numerical examples indicate that the formulae are easy to be implemented and accurate.
\end{abstract}

Keywords: contingent claims, high peak, heavy tails, volatility smile, overshoot.

\section{Introduction}

Many researches have been conducted to modify the Black-Scholes model based on Brownian motion and normal distribution in order to incorporate two empirical features: (1) The asymmetric leptokurtic features. In other words, the return distribution is skewed to the left, and has a higher peak and two heavier tails than those of the normal distribution. (2) The volatility smile. More precisely, if the Black-Scholes model is correct, then the implied volatility should be constant; but it is widely recognized that the implied volatility curve resembles a "smile," meaning it is a convex curve of the strike price.

* S. G. Kou, 312 Mudd Building, Department of IEOR, Columbia University, New York, NY 10027, e-mail: sk75@columbia.edu; Hui Wang, Division of Applied Mathematics, Brown University, Box F, Providence, RI 02912, e-mail: huiwang@cfm.brown.edu 
To incorporate the asymmetric leptokurtic features in asset pricing, a variety of models have been proposed, including, among others: (a) chaos theory, fractal Brownian motion, and stable processes; (b) generalized hyperbolic models, including log $t$ model and log hyperbolic model; (c) time changed Brownian motions, including log variance gamma model. In a parallel development, different models are also proposed to incorporate the "volatility smile" in option pricing. Popular ones are: (a) stochastic volatility ${ }^{1}$ and GARCH models; (b) constant elasticity model (CEV model); (c) normal jump diffusion models; (d) affine stochastic volatility and affine jump diffusion models; (e) models based on Lévy processes. For the background of these alternative models, see, for example, Hull (2000).

Unlike the original Black-Scholes model, although many alternative models can lead to analytic solutions for European call and put options, it is difficult to do so for path-dependent options, such as American options, lookback options, and barrier options. Even numerical methods for these derivatives are not easy. For example, the convergence rates of binomial trees and Monte Carlo simulation for path-dependent options are typically much slower than those for call and put options; for a survey, see, for example, Boyle, Broadie, and Glasserman (1997).

This paper attempts to extend the analytical tractability of Black-Scholes analysis for the classical geometric Brownian motion to alternative models with jumps. In particular, we demonstrate that a double exponential jump diffusion model (Kou, 2002) can lead to analytic approximation for finite horizon American options (by extending the approximation in Barone-Adesi and Whaley, 1987, for the classical geometric Brownian motion model), and analytical solutions for lookback, barrier, and perpetual American options.

The paper is organized as follows. Section 2 gives basic setting of the double exponential jump diffusion model, and presents intuition on why the analytical solutions are possible. An analytical approximation of finite time American options is given in Section 3, and the analysis of other path-dependent options is conducted in Section 4. The concluding remarks

\footnotetext{
${ }^{1}$ One empirical phenomenon worth mentioning is that the daily return distribution tends to have more kurtosis than the distribution of monthly returns. As Das and Foresi (1996) point out, this is consistent with models with jumps, but inconsistent with stochastic volatility models or other pure diffusion models.
} 
in given in Section 5. All the proofs are given in the appendices.

\section{Background and Intuition}

\subsection{The Double Exponential Jump Diffusion Model}

Under the double exponential jump diffusion model, the dynamics of the asset price $S(t)$ is given by

$$
\frac{d S(t)}{S(t-)}=\mu d t+\sigma d W(t)+d\left(\sum_{i=1}^{N(t)}\left(V_{i}-1\right)\right),
$$

where $W(t)$ is a standard Brownian motion, $N(t)$ a Poisson process with rate $\lambda$, and $\left\{V_{i}\right\}$ a sequence of independent identically distributed (i.i.d.) nonnegative random variables such that $Y=\log (V)$ has an asymmetric double exponential distribution with the density

$$
f_{Y}(y)=p \cdot \eta_{1} e^{-\eta_{1} y} \mathbf{1}_{\{y \geq 0\}}+q \cdot \eta_{2} e^{\eta_{2} y} \mathbf{1}_{\{y<0\}}, \quad \eta_{1}>1, \eta_{2}>0,
$$

where $p, q \geq 0, p+q=1$. Here the condition $\eta_{1}>1$ is imposed to ensure that the stock price $S(t)$ has finite expectation. Note that the means of the two exponential distributions are $1 / \eta_{1}$ and $1 / \eta_{2}$ respectively. In the model, all sources of randomness, $N(t), W(t)$, and $Y$ 's, are assumed to be independent.

Because of the jumps, the risk-neutral probability measure is not unique. Following Lucas (1978), Naik and Lee (1990), it can be shown (see, e.g., Kou 2002) that, by using the rational expectations argument with a HARA type utility function for the representative agent, one can choose a particular risk-neutral measure ${ }^{2} \mathrm{P}^{*}$ so that the equilibrium price of an option is given by the expectation under this risk neutral measure of the discounted option payoff. Under this risk neutral probability measure, the asset price $S(t)$ still follows a double exponential jump diffusion process ${ }^{3}$ :

$$
\frac{d S(t)}{S(t-)}=\left(r-\lambda^{*} \zeta^{*}\right) d t+\sigma d W^{*}(t)+d\left(\sum_{i=1}^{N^{*}(t)}\left(V_{i}^{*}-1\right)\right),
$$

\footnotetext{
${ }^{2}$ The measure $\mathrm{P}^{*}$ is called risk-neutral because that $\mathrm{E}^{*}\left(e^{-r t} S(t)\right)=S(0)$.

${ }^{3}$ For option pricing, the case of the underlying asset having a continuous dividend yield $\delta$ can be easily treated by changing $r$ to $r-\delta$ in (1).
} 
with the return process $X(t)=\log (S(t) / S(0))$ given by

$$
X(t)=\left(r-\frac{1}{2} \sigma^{2}-\lambda^{*} \zeta^{*}\right) t+\sigma W^{*}(t)+\sum_{i=1}^{N^{*}(t)} Y_{i}^{*}, \quad X(0)=0 .
$$

Here $W^{*}(t)$ is a standard Brownian motion under $\mathrm{P}^{*},\left\{N^{*}(t) ; t \geq 0\right\}$ is a Poisson process with intensity $\lambda^{*}, V^{*}=e^{Y^{*}}$. The $\log$ jump sizes $\left\{Y_{1}^{*}, Y_{2}^{*}, \cdots\right\}$ still form a sequence of independent identically distributed (i.i.d.) random variables with a new double exponential density $f_{Y^{*}}(y) \sim p^{*} \cdot \eta_{1}^{*} e^{-\eta_{1}^{*} y} \mathbf{1}_{\{y \geq 0\}}+q^{*} \cdot \eta_{2}^{*} e^{y \eta_{2}^{*}} \mathbf{1}_{\{y<0\}}$. The constants $p^{*}, q^{*} \geq 0, p^{*}+q^{*}=1$, $\lambda^{*}>0, \eta_{1}^{*}>1, \eta_{2}^{*}>0$, and

$$
\zeta^{*}:=\mathrm{E}^{*}\left[V^{*}\right]-1=\frac{p^{*} \eta_{1}^{*}}{\eta_{1}^{*}-1}+\frac{q^{*} \eta_{2}^{*}}{\eta_{2}^{*}+1}-1
$$

all depend on the utility function of the representative agent. All sources of randomness, $N^{*}(t), W^{*}(t)$, and $Y^{*}$ s, are still independent under $\mathrm{P}^{*}$.

Since we focus on option pricing in this paper, to simplify the notation (without causing much confusion), we shall drop the superscript $*$ in the parameters, i.e. using $p, q, \eta_{1}, \eta_{2}$ rather than $p^{*}, q^{*}, \eta_{1}^{*}, \eta_{2}^{*}$. The understanding is that all the processes and parameters below are under the risk-neutral probability measure $\mathrm{P}^{*}$.

\subsection{Intuition of the Pricing Formulae}

Without the jump part, the model simply becomes the classical geometric Brownian motion model. Pricing formulae for American options, barrier options, and lookback options are all well known under the geometric Brownian motion model ${ }^{4}$. With the jump part, however, it becomes very difficult to derive analytical solutions for these options.

The reason for that is as follows. To price American options, barrier options, and lookback options for general jump diffusion processes, it is crucial to study the first passage times that the process crosses a flat boundary with a level $b$. Without loss of generality, assume $b>0$. When a jump diffusion process crosses the boundary, sometimes it hits the boundary exactly

\footnotetext{
${ }^{4}$ Davydov and Linetsky $(2001,2003)$ provide analytical solutions for various path-dependent options under the CEV diffusion model.
} 
between the terminal state $X(t)$ and the overshoot $X\left(\tau_{b}\right)-b$ is not available ${ }^{6}$.

It is worth mentioning that the double exponential jump diffusion process is a special case of Lévy process with two-sided jumps, whose characteristic exponent admits the (unique) representation

$$
\phi(\theta)=\mathrm{E}\left[e^{i \theta X_{1}}\right]=\exp \left\{i \gamma \theta-\frac{1}{2} A \theta^{2}+\int_{-\infty}^{\infty}\left(e^{i \theta y}-1-i \theta y 1_{\{|y| \leq 1\}}\right) \Pi(d y)\right\},
$$

where the generating triplet $(\gamma, A, \Pi)$ is given by

$$
\begin{gathered}
A=\sigma^{2}, \quad \gamma=\mu+\lambda p\left(\frac{1-e^{-\eta_{1}}}{\eta_{1}}-e^{-\eta_{1}}\right)-\lambda q\left(\frac{1-e^{-\eta_{2}}}{\eta_{2}}-e^{-\eta_{2}}\right) \\
\Pi(d y)=\lambda \cdot f_{Y}(y) d y=\lambda p \eta_{1} e^{-\eta_{1} y} \mathbf{1}_{\{y \geq 0\}} d y+\lambda q \eta_{2} e^{\eta_{2} y} \mathbf{1}_{\{y<0\}} d y
\end{gathered}
$$

If the jump size distribution is one-sided, one can solve the overshoot problems ${ }^{7}$ by either using renewal equations or fluctuation identities for Lévy processes; see, e.g., Avram, Chan, and Usabel (2001), Rogers (2000). However, for two-sided jumps, because of the ladder-variable problems, generally speaking the renewal equations are not available and the fluctuation identities becomes too complicated for explicit computation; see, e.g., the discussion in Siegmund (1985) and Rogers (2000).

\subsection{Some Notations}

The moment generating function of $X(t)$ is given by $\mathrm{E}^{*}\left[e^{\theta X(t)}\right]=\exp \{G(\theta) t\}$, where the function $G(\cdot)$ is defined as

$$
G(x):=x\left(r-\frac{1}{2} \sigma^{2}-\lambda \zeta\right)+\frac{1}{2} x^{2} \sigma^{2}+\lambda\left(\frac{p \eta_{1}}{\eta_{1}-x}+\frac{q \eta_{2}}{\eta_{2}+x}-1\right) .
$$

Lemma 3.1 in Kou and Wang (2003) shows that the equation $G(x)=\alpha, \forall \alpha>0$, has exactly four roots: $\beta_{1, \alpha}, \beta_{2, \alpha},-\beta_{3, \alpha},-\beta_{4, \alpha}$, where

$$
0<\beta_{1, \alpha}<\eta_{1}<\beta_{2, \alpha}<\infty, \quad 0<\beta_{3, \alpha}<\eta_{2}<\beta_{4, \alpha}<\infty .
$$

\footnotetext{
${ }^{6}$ See, for example, Siegmund (1985), Boyarchenko and Levendorskii (2002b), and Kyprianou and Pistorius (2003) for some representations (though not explicit calculations) related to the overshoot problems for general Lévy processes.

${ }^{7}$ For a jump diffusion process with one-sided jumps, the overshoot problem may not occur if the boundary level is on the opposite direction of the jumps; e.g., the jump sizes are negative and the boundary level $b>0$. Under this circumstance, explicit solution may be possible (at least for perpetual American options) even if the distribution of jump size take a general form; see e.g. Mordecki (1999).
} 
To use the Itô formula for jump processes, we also need the infinitesimal generator of $X(t)$ :

$$
(\mathcal{L} V)(x):=\frac{1}{2} \sigma^{2} V^{\prime \prime}(x)+\left(r-\frac{1}{2} \sigma^{2}-\lambda \zeta\right) V^{\prime}(x)+\lambda \int_{-\infty}^{\infty}[V(x+y)-V(x)] f_{Y}(y) d y .
$$

\section{Pricing Finite Time Horizon American Options}

Most of call and put options traded in the exchanges in both U.S. and Europe are American type options. Therefore, it is of great interest to calculate the prices of American options accurately and quickly. The price of a finite horizon American option is the solution of a finite horizon free boundary problem. Even within the classical geometric Brownian motion model, except in the case of the American call option with no dividend, there is no analytical solution available . $^{8}$

To price American options under general jump diffusion models, one may consider numerically solving the free boundary problems via lattice or differential equation methods; see, e.g., Amin (1993), Zhang (1997), d'Halluin, Forsyth, and Vetzal (2003). Extending the Barone-Adesi and Whaley (1987) approximation for the classical geometric Brownian motion model, we shall consider an alternative approach that takes into consideration of the special structure of the double exponential jump diffusions. One motivation for such an extension is its simplicity, as it yields an analytic approximation that only involves the price of a European option. Our numerical results in Tables 1 and 2 suggest that the approximation error is typically less than $2 \%$, which is less than the typical bid-ask spread (about $5 \%$ to $10 \%$ ) for American options in exchanges. Therefore, the approximation can serve as an easy way to get a quick estimate that is perhaps accurate enough for many practical situations.

The extension of Barone-Adesi and Whaley's method works nicely for double exponential jump diffusion models mainly because explicit solutions are available to a class of relevant integro-differential free boundary problems; see (15) and (16). We want to point out that

\footnotetext{
${ }^{8}$ For recent developments of numerical solution and analytic approximation of finite horizon American options within the classical geometric Brownian motion model, see, for example, Broadie and Detemple (1996), Carr (1998), Ju (1998), Geske and Johnson (1984), McMillan (1986), Tilley (1993), Tsitsiklis and van Roy (1999), Sullivan (2000), Broadie and Glasserman (1997), Carriére (1996), Longstaff and Schwartz (2001), Rogers (2002), Haugh and Kogan (2002) and references therein.
} 
there exist other more elaborate but more accurate approximations (such as Broadie and Detemple 1996, Carr 1998, and Ju 1998) for geometric Brownian motion models, and whether these algorithms can be effectively extended to jump diffusion models invites further investigation.

To simplify notation, we shall focus only on the finite horizon American put option without dividends, as the methodology is also valid for the finite horizon American call option with dividends. The analytic approximation involves two quantities, $\operatorname{EuP}(v, t)$ which denotes the price of the European put option with initial stock price $v$ and maturity $t$, and $\mathrm{P}^{v}[S(t) \leq K]$ which is the probability that the stock price at $t$ is below $K$ with initial stock price $v$. Both $\operatorname{EuP}(v, t)$ and $\mathrm{P}^{v}[S(t) \leq K]$ can be computed fast by using either the closed form solutions in Kou (2002) or the Laplace transforms in Petrella, Kou, and Wang (2003).

We need some notations. Let $z=1-e^{-r t}, \beta_{3} \equiv \beta_{3, \frac{r}{z}}, \beta_{4} \equiv \beta_{4, \frac{r}{z}}, C_{\beta}=\beta_{3} \beta_{4}\left(1+\eta_{2}\right)$, $D_{\beta}=\eta_{2}\left(1+\beta_{3}\right)\left(1+\beta_{4}\right)$, in the notation of equation (4). Define $v_{0} \equiv v_{0}(t) \in(0, K)$ as the unique solution ${ }^{9}$ to the equation

$$
C_{\beta} K-D_{\beta}\left[v_{0}+\operatorname{EuP}\left(v_{0}, t\right)\right]=\left(C_{\beta}-D_{\beta}\right) K e^{-r t} \cdot \mathrm{P}^{v_{0}}[S(t) \leq K]
$$

Note that the left hand side of $(6)$ is a strictly decreasing function of $v_{0}$ (because $v_{0}+$ $\left.\operatorname{EuP}\left(v_{0}, t\right)=e^{-r t} \mathrm{E}^{*}\left[\max (S(t), K) \mid S(0)=v_{0}\right]\right)$, and the right hand side of (6) is a strictly increasing function of $v_{0}$ (because $\left.C_{\beta}-D_{\beta}=\beta_{3} \beta_{4}-\eta_{2}\left(1+\beta_{3}+\beta_{4}\right)<0\right)$. Therefore, $v_{0}$ can be obtained easily by using, for example, the bisection method.

Approximation: The price of a finite horizon American put option with maturity $t$ and strike $K$ can be approximated by $\psi(S(0), t)$, where the value function $\psi$ is given by

$$
\psi(v, t)=\left\{\begin{array}{ll}
\operatorname{EuP}(v, t)+A v^{-\beta_{3}}+B v^{-\beta_{4}} ; & \text { if } v \geq v_{0} \\
K-v ; & \text { if } v \leq v_{0}
\end{array},\right.
$$

with $v_{0}$ being the unique root of the equation (6) and the two constants $A$ and $B$ given by

$$
A=\frac{v_{0}^{\beta_{3}}}{\beta_{4}-\beta_{3}}\left\{\beta_{4} K-\left(1+\beta_{4}\right)\left[v_{0}+\operatorname{EuP}\left(v_{0}, t\right)\right]+K e^{-r t} \mathrm{P}^{v_{0}}[S(t) \leq K]\right\}>0
$$

\footnotetext{
${ }^{9}$ In Appendix A, we give a better upper bound in (18) for $v_{0}$, that is $K>v_{0}+\operatorname{EuP}\left(v_{0}, t\right)$.
} 


$$
B=\frac{v_{0}^{\beta_{4}}}{\beta_{3}-\beta_{4}}\left\{\beta_{3} K-\left(1+\beta_{3}\right)\left[v_{0}+\operatorname{EuP}\left(v_{0}, t\right)\right]+K e^{-r t} \mathrm{P}^{v_{0}}[S(t) \leq K]\right\}>0 .
$$

Tables 1 and 2 present numerical results for finite horizon American put options, corresponding to $t=0.25$ and $t=1.0$ years. The paremeters used here are $S(0)=100, p=0.6$, $r=0.05$. To save space, the numerical results for $t=0.5$ and $t=1.5$ are omitted, which can be obstained from the authors upon request. We choose this set of maturities, because most of the American options traded in exchanges have maturities between three months and one year. The "true" value is calculated by using the enhanced binomial tree method as in Amin (1993) with 1600 steps (to ensure that the accuracy is up to about a penny) and the two-point Richardson extrapolation for the square-root convergence rate.

In the tables the maximum relative error is only about $2.6 \%$, while in most cases the relative errors are below 1\%. Note also the approximation tends to works better for small maturity $t$; this is because of the assumption (14) in the approximation, as will be explained in Appendix A.1. All the calculations are conducted on a Pentium 1500 PC. The approximation runs very fast, taking only about 0.04 second to compute one price, irrespective to the parameter ranges; while the lattice method works much slower, taking about over one hour to compute one price.

\section{Pricing Other Path-Dependent Options}

Lookback and barrier options are among the most popular path-dependent options traded in exchanges worldwide and also in over-the-counter markets; and perpetual American options are interesting because they serve as simple examples to illustrate finance theory ${ }^{10}$. We shall

\footnotetext{
${ }^{10}$ Pricing of barrier, lookback, and perpetual American options also arises quite often in other contexts. For example, Merton (1974), Black and Cox (1976), and more recently Leland (1994), Longstaff (1996), Longstaff and Schwartz (1995), among others, have used lookback and barrier options to value debt and contingent claims in corporate finance with endogenous default; McDonald and Siegel (1985) use perpetual American options in studying real options. Within the classical geometric Brownian motion framework, closed form solutions for lookback, barrier, and perpetual American options are available, at least since McKean (1965), Merton (1973), Goldman, Sosin, and Gatto (1979), Conze and Viswanathan (1991).
} 


\begin{tabular}{|c|c|c|c|c|c|c|c|c|c|c|}
\hline \multicolumn{5}{|c|}{ Parameter Values } & \multirow{2}{*}{$\begin{array}{l}\text { "True" Value } \\
\text { (a) }\end{array}$} & \multirow{2}{*}{$\begin{array}{l}\text { CPU } \\
\text { Time }\end{array}$} & \multirow{2}{*}{$\begin{array}{l}\text { Approx. } \\
\text { (b) }\end{array}$} & \multirow{2}{*}{$\begin{array}{l}\text { CPU } \\
\text { Time }\end{array}$} & \multirow{2}{*}{$\begin{array}{l}\text { Abs. Error } \\
\text { (b)-(a) }\end{array}$} & \multirow{2}{*}{$\begin{array}{l}\text { Relative Error } \\
\text { ((b)-(a))/(a) }\end{array}$} \\
\hline$K$ & $\sigma$ & $\lambda$ & $\eta_{1}$ & $\eta_{2}$ & & & & & & \\
\hline 110 & 0.2 & 3 & 25 & 25 & 10.48 & 4030 & 10.43 & 0.03 & -0.05 & $-0.5 \%$ \\
\hline 110 & 0.2 & 3 & 25 & 50 & 10.42 & 4029 & 10.38 & 0.04 & -0.04 & $-0.4 \%$ \\
\hline 110 & 0.2 & 3 & 50 & 25 & 10.36 & 4029 & 10.31 & 0.03 & -0.05 & $-0.5 \%$ \\
\hline 110 & 0.2 & 3 & 50 & 50 & 10.31 & 4027 & 10.26 & 0.04 & -0.05 & $-0.5 \%$ \\
\hline 110 & 0.2 & 7 & 25 & 25 & 10.81 & 4030 & 10.79 & 0.03 & -0.02 & $-0.2 \%$ \\
\hline 110 & 0.2 & 7 & 25 & 50 & 10.68 & 4029 & 10.64 & 0.04 & -0.04 & $-0.4 \%$ \\
\hline 110 & 0.2 & 7 & 50 & 25 & 10.51 & 4028 & 10.47 & 0.03 & -0.04 & $-0.4 \%$ \\
\hline 110 & 0.2 & 7 & 50 & 50 & 10.39 & 4027 & 10.34 & 0.03 & -0.05 & $-0.5 \%$ \\
\hline 110 & 0.3 & 3 & 25 & 25 & 11.90 & 4023 & 11.86 & 0.04 & -0.04 & $-0.3 \%$ \\
\hline 110 & 0.3 & 3 & 25 & 50 & 11.84 & 4021 & 11.79 & 0.03 & -0.05 & $-0.4 \%$ \\
\hline 110 & 0.3 & 3 & 50 & 25 & 11.78 & 4025 & 11.73 & 0.03 & -0.05 & $-0.4 \%$ \\
\hline 110 & 0.3 & 3 & 50 & 50 & 11.72 & 4028 & 11.67 & 0.03 & -0.05 & $-0.4 \%$ \\
\hline 110 & 0.3 & 7 & 25 & 25 & 12.23 & 4023 & 12.19 & 0.04 & -0.04 & $-0.3 \%$ \\
\hline 110 & 0.3 & 7 & 25 & 50 & 12.09 & 4020 & 12.05 & 0.03 & -0.04 & $-0.3 \%$ \\
\hline 110 & 0.3 & 7 & 50 & 25 & 11.94 & 4025 & 11.90 & 0.04 & -0.04 & $-0.3 \%$ \\
\hline 110 & 0.3 & 7 & 50 & 50 & 11.80 & 4026 & 11.75 & 0.03 & -0.05 & $-0.4 \%$ \\
\hline 100 & 0.2 & 3 & 25 & 25 & $\begin{array}{l}3.78 \\
\end{array}$ & 4038 & $\begin{array}{l}3.78 \\
\end{array}$ & 0.03 & 0.00 & $0.0 \%$ \\
\hline 100 & 0.2 & 3 & 25 & 50 & 3.66 & 4043 & 3.66 & 0.04 & 0.00 & $0.0 \%$ \\
\hline 100 & 0.2 & 3 & 50 & 25 & 3.62 & 4036 & 3.62 & 0.03 & 0.00 & $0.0 \%$ \\
\hline 100 & 0.2 & 3 & 50 & 50 & 3.50 & 4042 & 3.50 & 0.03 & 0.00 & $0.0 \%$ \\
\hline 100 & 0.2 & 7 & 25 & 25 & 4.26 & 4034 & 4.27 & 0.03 & 0.01 & $0.2 \%$ \\
\hline 100 & 0.2 & 7 & 25 & 50 & 4.01 & 4038 & 4.02 & 0.03 & 0.01 & $0.2 \%$ \\
\hline 100 & 0.2 & 7 & 50 & 25 & 3.91 & 4042 & 3.91 & 0.04 & 0.00 & $0.0 \%$ \\
\hline 100 & 0.2 & 7 & 50 & 50 & 3.64 & 4042 & 3.64 & 0.03 & 0.00 & $0.0 \%$ \\
\hline 100 & 0.3 & 3 & 25 & 25 & 5.63 & 4037 & 5.62 & 0.03 & -0.01 & $-0.2 \%$ \\
\hline 100 & 0.3 & 3 & 25 & 50 & 5.55 & 4035 & 5.54 & 0.04 & -0.01 & $-0.2 \%$ \\
\hline 100 & 0.3 & 3 & 50 & 25 & 5.50 & 4040 & 5.50 & 0.03 & 0.00 & $0.0 \%$ \\
\hline 100 & 0.3 & 3 & 50 & 50 & 5.42 & 4042 & 5.41 & 0.03 & -0.01 & $-0.2 \%$ \\
\hline 100 & 0.3 & 7 & 25 & 25 & 5.99 & 4038 & 5.99 & 0.04 & 0.00 & $0.0 \%$ \\
\hline 100 & 0.3 & 7 & 25 & 50 & 5.81 & 4035 & 5.81 & 0.03 & 0.00 & $0.0 \%$ \\
\hline 100 & 0.3 & 7 & 50 & 25 & 5.71 & 4040 & 5.71 & 0.03 & 0.00 & $0.0 \%$ \\
\hline 100 & 0.3 & 7 & 50 & 50 & 5.52 & 4041 & 5.51 & 0.04 & -0.01 & $-0.2 \%$ \\
\hline 90 & 0.2 & 3 & 25 & 25 & 0.75 & 4033 & 0.76 & 0.03 & 0.01 & $1.3 \%$ \\
\hline 90 & 0.2 & 3 & 25 & 50 & 0.65 & 4031 & 0.66 & 0.04 & 0.01 & $1.5 \%$ \\
\hline 90 & 0.2 & 3 & 50 & 25 & 0.68 & 4031 & 0.69 & 0.04 & 0.01 & $1.5 \%$ \\
\hline 90 & 0.2 & 3 & 50 & 50 & 0.59 & 4029 & 0.60 & 0.04 & 0.01 & $1.7 \%$ \\
\hline 90 & 0.2 & 7 & 25 & 25 & 1.03 & 4033 & 1.04 & 0.03 & 0.01 & $1.0 \%$ \\
\hline 90 & 0.2 & 7 & 25 & 50 & 0.82 & 4031 & 0.83 & 0.04 & 0.01 & $1.2 \%$ \\
\hline 90 & 0.2 & 7 & 50 & 25 & 0.87 & 4030 & 0.88 & 0.03 & 0.01 & $1.1 \%$ \\
\hline 90 & 0.2 & 7 & 50 & 50 & 0.66 & 4029 & 0.67 & 0.03 & 0.01 & $1.5 \%$ \\
\hline 90 & 0.3 & 3 & 25 & 25 & 1.92 & 4025 & 1.93 & 0.04 & 0.01 & $0.5 \%$ \\
\hline 90 & 0.3 & 3 & 25 & 50 & 1.85 & 4024 & 1.86 & 0.03 & 0.01 & $0.5 \%$ \\
\hline 90 & 0.3 & 3 & 50 & 25 & 1.84 & 4027 & 1.85 & 0.03 & 0.01 & $0.5 \%$ \\
\hline 90 & 0.3 & 3 & 50 & 50 & 1.77 & 4030 & 1.78 & 0.04 & 0.01 & $0.6 \%$ \\
\hline 90 & 0.3 & 7 & 25 & 25 & 2.19 & 4025 & 2.20 & 0.03 & 0.01 & $0.5 \%$ \\
\hline 90 & 0.3 & 7 & 25 & 50 & 2.03 & 4023 & 2.03 & 0.03 & 0.00 & $0.0 \%$ \\
\hline 90 & 0.3 & 7 & 50 & 25 & 2.01 & 4028 & 2.02 & 0.04 & 0.01 & $0.5 \%$ \\
\hline 90 & 0.3 & 7 & 50 & 50 & 1.84 & 4028 & 1.85 & 0.03 & 0.01 & $0.5 \%$ \\
\hline
\end{tabular}

Table 1: Comparison of the approximation and the true value for finite horizon American put option with $t=0.25$ year. The CPU times are in seconds. 


\begin{tabular}{|c|c|c|c|c|c|c|c|c|c|c|}
\hline \multicolumn{5}{|c|}{ Parameter Values } & \multirow{2}{*}{$\begin{array}{l}\text { "True" Value } \\
\text { (a) }\end{array}$} & \multirow{2}{*}{$\begin{array}{l}\text { CPU } \\
\text { Time }\end{array}$} & \multirow{2}{*}{$\begin{array}{l}\text { Approx. } \\
\text { (b) }\end{array}$} & \multirow{2}{*}{$\begin{array}{l}\text { CPU } \\
\text { Time }\end{array}$} & \multirow{2}{*}{$\begin{array}{l}\text { Abs. Error } \\
\text { (b)-(a) }\end{array}$} & \multirow{2}{*}{$\begin{array}{l}\text { Relative Error } \\
\text { ((b)-(a))/(a) }\end{array}$} \\
\hline$K$ & $\sigma$ & $\lambda$ & $\eta_{1}$ & $\eta_{2}$ & & & & & & \\
\hline 110 & 0.2 & 3 & 25 & 25 & 12.37 & 4026 & 12.32 & 0.04 & -0.05 & $-0.4 \%$ \\
\hline 110 & 0.2 & 3 & 25 & 50 & 12.17 & 4026 & 12.11 & 0.03 & -0.06 & $-0.5 \%$ \\
\hline 110 & 0.2 & 3 & 50 & 25 & 12.04 & 4025 & 12.00 & 0.04 & -0.04 & $-0.3 \%$ \\
\hline 110 & 0.2 & 3 & 50 & 50 & 11.84 & 4025 & 11.78 & 0.03 & -0.06 & $-0.5 \%$ \\
\hline 110 & 0.2 & 7 & 25 & 25 & 13.29 & 4026 & 13.27 & 0.03 & -0.02 & $-0.2 \%$ \\
\hline 110 & 0.2 & 7 & 25 & 50 & 12.85 & 4026 & 12.79 & 0.04 & -0.06 & $-0.5 \%$ \\
\hline 110 & 0.2 & 7 & 50 & 25 & 12.54 & 4024 & 12.54 & 0.03 & 0.00 & $0.0 \%$ \\
\hline 110 & 0.2 & 7 & 50 & 50 & 12.08 & 4024 & 12.03 & 0.03 & -0.05 & $-0.4 \%$ \\
\hline 110 & 0.3 & 3 & 25 & 25 & 15.79 & 4025 & 15.76 & 0.04 & -0.03 & $-0.2 \%$ \\
\hline 110 & 0.3 & 3 & 25 & 50 & 15.63 & 4027 & 15.59 & 0.04 & -0.04 & $-0.3 \%$ \\
\hline 110 & 0.3 & 3 & 50 & 25 & 15.51 & 4028 & 15.49 & 0.03 & -0.02 & $-0.1 \%$ \\
\hline 110 & 0.3 & 3 & 50 & 50 & 15.36 & 4028 & 15.32 & 0.03 & -0.04 & $-0.3 \%$ \\
\hline 110 & 0.3 & 7 & 25 & 25 & 16.51 & 4025 & 16.51 & 0.04 & 0.00 & $0.0 \%$ \\
\hline 110 & 0.3 & 7 & 25 & 50 & 16.17 & 4028 & 16.14 & 0.03 & -0.03 & $-0.2 \%$ \\
\hline 110 & 0.3 & 7 & 50 & 25 & 15.89 & 4028 & 15.91 & 0.03 & 0.02 & $0.1 \%$ \\
\hline 110 & 0.3 & 7 & 50 & 50 & 15.53 & 4028 & 15.52 & 0.04 & -0.01 & $-0.1 \%$ \\
\hline 100 & 0.2 & 3 & 25 & 25 & 6.60 & 4027 & $\begin{array}{l}6.62 \\
\end{array}$ & 0.03 & 0.02 & $0.3 \%$ \\
\hline 100 & 0.2 & 3 & 25 & 50 & 6.36 & 4025 & 6.37 & 0.03 & 0.01 & $0.2 \%$ \\
\hline 100 & 0.2 & 3 & 50 & 25 & 6.26 & 4025 & 6.29 & 0.04 & 0.03 & $0.5 \%$ \\
\hline 100 & 0.2 & 3 & 50 & 50 & 6.01 & 4025 & 6.03 & 0.03 & 0.02 & $0.3 \%$ \\
\hline 100 & 0.2 & 7 & 25 & 25 & 7.57 & 4026 & 7.62 & 0.03 & 0.05 & $0.7 \%$ \\
\hline 100 & 0.2 & 7 & 25 & 50 & 7.07 & 4026 & 7.09 & 0.04 & 0.02 & $0.3 \%$ \\
\hline 100 & 0.2 & 7 & 50 & 25 & 6.83 & 4024 & 6.88 & 0.03 & 0.05 & $0.7 \%$ \\
\hline 100 & 0.2 & 7 & 50 & 50 & 6.28 & 4025 & 6.31 & 0.04 & 0.03 & $0.5 \%$ \\
\hline 100 & 0.3 & 3 & 25 & 25 & 10.10 & 4025 & 10.13 & 0.03 & 0.03 & $0.3 \%$ \\
\hline 100 & 0.3 & 3 & 25 & 50 & 9.94 & 4027 & 9.96 & 0.03 & 0.02 & $0.2 \%$ \\
\hline 100 & 0.3 & 3 & 50 & 25 & 9.83 & 4028 & 9.87 & 0.04 & 0.04 & $0.4 \%$ \\
\hline 100 & 0.3 & 3 & 50 & 50 & 9.67 & 4029 & 9.70 & 0.03 & 0.03 & $0.3 \%$ \\
\hline 100 & 0.3 & 7 & 25 & 25 & 10.81 & 4025 & 10.86 & 0.03 & 0.05 & $0.5 \%$ \\
\hline 100 & 0.3 & 7 & 25 & 50 & 10.46 & 4026 & 10.49 & 0.03 & 0.03 & $0.3 \%$ \\
\hline 100 & 0.3 & 7 & 50 & 25 & 10.22 & 4036 & 10.29 & 0.04 & 0.07 & $0.7 \%$ \\
\hline 100 & 0.3 & 7 & 50 & 50 & 9.85 & 4028 & 9.89 & 0.02 & 0.04 & $0.4 \%$ \\
\hline 90 & 0.2 & 3 & 25 & 25 & 2.91 & 4025 & 2.96 & 0.04 & 0.05 & $1.7 \%$ \\
\hline 90 & 0.2 & 3 & 25 & 50 & 2.70 & 4025 & 2.75 & 0.03 & 0.05 & $1.9 \%$ \\
\hline 90 & 0.2 & 3 & 50 & 25 & 2.66 & 4025 & 2.72 & 0.04 & 0.06 & $2.3 \%$ \\
\hline 90 & 0.2 & 3 & 50 & 50 & 2.46 & 4025 & 2.51 & 0.03 & 0.05 & $2.0 \%$ \\
\hline 90 & 0.2 & 7 & 25 & 25 & 3.68 & 4026 & 3.75 & 0.03 & 0.07 & $1.9 \%$ \\
\hline 90 & 0.2 & 7 & 25 & 50 & 3.24 & 4025 & 3.29 & 0.04 & 0.05 & $1.5 \%$ \\
\hline 90 & 0.2 & 7 & 50 & 25 & 3.12 & 4024 & 3.20 & 0.03 & 0.08 & $2.6 \%$ \\
\hline 90 & 0.2 & 7 & 50 & 50 & 2.66 & 4025 & 2.72 & 0.03 & 0.06 & $2.3 \%$ \\
\hline 90 & 0.3 & 3 & 25 & 25 & 5.79 & 4024 & 5.85 & 0.04 & 0.06 & $1.0 \%$ \\
\hline 90 & 0.3 & 3 & 25 & 50 & 5.65 & 4025 & 5.70 & 0.03 & 0.05 & $0.9 \%$ \\
\hline 90 & 0.3 & 3 & 50 & 25 & 5.58 & 4028 & 5.64 & 0.04 & 0.06 & $1.1 \%$ \\
\hline 90 & 0.3 & 3 & 50 & 50 & 5.43 & 4029 & 5.49 & 0.03 & 0.06 & $1.1 \%$ \\
\hline 90 & 0.3 & 7 & 25 & 25 & 6.42 & 4027 & 6.49 & 0.04 & 0.07 & $1.1 \%$ \\
\hline 90 & 0.3 & 7 & 25 & 50 & 6.09 & 4026 & 6.15 & 0.03 & 0.06 & $1.0 \%$ \\
\hline 90 & 0.3 & 7 & 50 & 25 & 5.92 & 4029 & 6.00 & 0.03 & 0.08 & $1.4 \%$ \\
\hline 90 & 0.3 & 7 & 50 & 50 & 5.59 & 4031 & 5.65 & 0.04 & 0.06 & $1.1 \%$ \\
\hline
\end{tabular}

Table 2: Comparison of the approximation and the true value for finite horizon American put option with $t=1$ year. 
demonstrate in this section that in the double exponential jump diffusion model the closed form solutions for these options can still be obtained.

\subsection{Lookback Options}

As the calculation for the lookback call option follows just by symmetry, we will only provide the result for the lookback put option, whose price is given by

$\mathrm{LP}(T)=\mathrm{E}^{*}\left[e^{-r T}\left(\max \left\{M, \max _{0 \leq t \leq T} S(t)\right\}-S(T)\right)\right]=\mathrm{E}^{*}\left[e^{-r T}\left(\max \left\{M, \max _{0 \leq t \leq T} S(t)\right\}\right)\right]-S(0)$, where $M \geq S(0)$ is a fixed constant representing the prefixed maximum at time 0 .

Theorem 4.1. Using the notation $\beta_{1, \alpha+r}$ and $\beta_{2, \alpha+r}$ as in (4), the Laplace transform of the lookback put is given by

$$
\int_{0}^{\infty} e^{-\alpha T} \operatorname{LP}(T) d T=\frac{S(0) A_{\alpha}}{C_{\alpha}}\left(\frac{S(0)}{M}\right)^{\beta_{1, \alpha+r}-1}+\frac{S(0) B_{\alpha}}{C_{\alpha}}\left(\frac{S(0)}{M}\right)^{\beta_{2, \alpha+r}-1}+\frac{M}{\alpha+r}-\frac{S(0)}{\alpha}
$$

for all $\alpha>0$; here

$$
A_{\alpha}=\frac{\left(\eta_{1}-\beta_{1, \alpha+r}\right) \beta_{2, \alpha+r}}{\beta_{1, \alpha+r}-1}, B_{\alpha}=\frac{\left(\beta_{2, \alpha+r}-\eta_{1}\right) \beta_{1, \alpha+r}}{\beta_{2, \alpha+r}-1}, C_{\alpha}=(\alpha+r) \eta_{1}\left(\beta_{2, \alpha+r}-\beta_{1, \alpha+r}\right) .
$$

The proof of Theorem 4.1 will be given in Appendix B.1. Essentially, the proof explores a link between the Laplace transform of the lookback option and the Laplace transform of the first passage times of the double exponential jump diffusion process as solved explicitly in Kou and Wang (2003).

\subsection{Barrier Options}

There are eight types of (one dimensional, single) barrier options, namely up (down)-and-in (out) call (put) options. For example, the price of a down-and-out put (DOP) option is given by $\mathrm{DOP}=\mathrm{E}^{*}\left[e^{-r T}(K-S(T))^{+} \mathbf{1}_{\left\{\min _{0 \leq t \leq T} S(t) \geq H\right\}}\right]$, where $H<S(0)$ is the barrier level. Since all the eight types barrier options can be solved in similar ways, we shall only illustrate with the up-and-in call (UIC) option, whose price is given by

$$
\mathrm{UIC}=\mathrm{E}^{*}\left[e^{-r T}(S(T)-K)^{+} \mathbf{1}_{\left\{\max _{0 \leq t \leq T} S(t) \geq H\right\}}\right]
$$


where $H>S(0)$ is the barrier level. Introduce the following notation: for any given probability $P$,

$$
\Psi\left(\mu, \sigma, \lambda, p, \eta_{1}, \eta_{2} ; a, b, T\right):=\mathrm{P}\left[Z(T) \geq a, \max _{0 \leq t \leq T} Z(t) \geq b\right]
$$

where under $\mathrm{P}, Z(t)$ is a double exponential jump diffusion process with drift $\mu$, volatility $\sigma$, and jump rate $\lambda$, i.e. $Z(t)=\mu t+\sigma W(t)+\sum_{i=1}^{N(t)} Y_{i}$, and $Y$ has a double exponential distribution with density $f_{Y}(y) \sim p \cdot \eta_{1} e^{-\eta_{1} y} \mathbf{1}_{\{y \geq 0\}}+q \cdot \eta_{2} e^{y \eta_{2}} \mathbf{1}_{\{y<0\}}$. The formula of the up-and-in call option will be written in terms of $\Psi$. The Laplace transforms of $\Psi$ is computed explicitly in Kou and Wang (2003).

Theorem 4.2 The price of the up-and-in call option is obtained as

$$
\begin{aligned}
\mathrm{UIC}=S(0) \Psi & \left(r+\frac{1}{2} \sigma^{2}-\lambda \zeta, \sigma, \tilde{\lambda}, \tilde{p}, \tilde{\eta}_{1}, \tilde{\eta}_{2} ; \log (K / S(0)), \log (H / S(0)), T\right) \\
& -K e^{-r T} \cdot \Psi\left(r-\frac{1}{2} \sigma^{2}-\lambda \zeta, \sigma, \lambda, p, \eta_{1}, \eta_{2} ; \log (K / S(0)), \log (H / S(0)), T\right)
\end{aligned}
$$

where $\tilde{p}=\frac{p}{1+\zeta} \cdot \frac{\eta_{1}}{\eta_{1}-1}, \tilde{\eta}_{1}=\eta_{1}-1, \quad \tilde{\eta}_{2}=\eta_{2}+1, \tilde{\lambda}=\lambda(\zeta+1)$, with $\zeta$ given in (3) and $\Psi$ in (10).

The proof of Theorem 4.2 will be given in Appendix B.2. It uses a change of numeraire argument, which intuitively change the unit of the money from the money market account to the underlying asset $S(t)$, to reduce the computation of the expectation to the difference of two probabilities. For further background of the change of numeraire argument for jump diffusion processes, see, for example, Schroder (1999).

\subsection{Numerical Results for Barrier and Lookback Options}

Since the solutions for barrier and lookback options are given in terms of Laplace transforms, numerical inversion of Laplace transforms becomes necessary. To do this, we shall use the Gaver-Stehfest algorithm. Given the Laplace transform function $\hat{f}(\alpha)=\int_{0}^{\infty} e^{-\alpha x} f(x) d x$ of a function $f(x)$, the algorithm generates a sequence $f_{n}(x)$ such that $f_{n}(x) \rightarrow f(x), n \rightarrow \infty$. The algorithm ${ }^{11}$ converges very fast; as we will see it typically converges nicely even for $n$

\footnotetext{
${ }^{11}$ The main advantages of the Gaver-Stehfest algorithm are: simplicity (a very short code will do the job), fast convergence, and good stability (i.e. the final output is not sensitive to a small perturbation of
} 


\begin{tabular}{|c|c|c|c|c|c|}
\hline \multicolumn{2}{|l|}{$n$} & \multicolumn{2}{|c|}{ Lookback Put } & \multicolumn{2}{|c|}{ Up-and-In Call } \\
\hline & & $\lambda=0.01$ & $\lambda=3$ & $\lambda=0.01$ & $\lambda=3$ \\
\hline 1 & & 17.20214 & 18.58516 & 10.32186 & 10.98416 \\
\hline 2 & & 16.55458 & 17.83041 & 9.81060 & 10.62657 \\
\hline 3 & & 16.14481 & 17.35415 & 9.49290 & 10.31446 \\
\hline 4 & & 15.95166 & 17.13035 & 9.34838 & 10.13851 \\
\hline 5 & & 15.87823 & 17.04556 & 9.29672 & 10.07120 \\
\hline 6 & & 15.85473 & 17.01851 & 9.28173 & 10.05461 \\
\hline 7 & & 15.84823 & 17.01105 & 9.27813 & 10.05280 \\
\hline 8 & & 15.84664 & 17.00923 & 9.27740 & 10.05309 \\
\hline 9 & & 15.84629 & 17.00884 & 9.27726 & 10.05315 \\
\hline 10 & & 15.84622 & 17.00877 & 9.27724 & 10.05307 \\
\hline Total CPU & ime & $0.541 \mathrm{sec}$ & $0.711 \mathrm{sec}$ & $2.849 \min$ & $2.815 \min$ \\
\hline Brownian Motic & 1 Case & 15.84226 & N.A. & 9.27451 & N.A. \\
\hline \multicolumn{6}{|c|}{ Monte Carlo Simulation } \\
\hline $\begin{array}{c}200 \text { Points } \\
\text { CPU Time: } 8 \text { min }\end{array}$ & $\begin{array}{l}\text { point est. } \\
95 \% \text { C.I. }\end{array}$ & $\begin{array}{c}15.39 \\
(15.22,15.56) \\
\end{array}$ & $\begin{array}{c}16.29 \\
(16.06,16.52) \\
\end{array}$ & $\begin{array}{c}9.14 \\
(8.90,9.38) \\
\end{array}$ & $\begin{array}{c}9.82 \\
(9.56,10.08) \\
\end{array}$ \\
\hline $\begin{array}{c}2000 \text { Points } \\
\text { CPU Time: } 37 \mathrm{~min}\end{array}$ & $\begin{array}{l}\text { point est. } \\
95 \% \text { C.I. }\end{array}$ & $\begin{array}{c}15.65 \\
(15.47,15.83)\end{array}$ & $\begin{array}{c}16.78 \\
(16.59,16.97)\end{array}$ & $\begin{array}{c}9.24 \\
(9.00,9.48)\end{array}$ & $\begin{array}{c}10.05 \\
(9.79,10.31)\end{array}$ \\
\hline
\end{tabular}

Table 3: The prices of lookback put and up-and-in call options. The Monte Carlo results are based on 16,000 simulation runs.

between 5 and 10. The details of implementation is reported in Kou and Wang (2001).

As a numerical illustration, we calculate both the lookback put option and the UIC barrier option in Table 3. For the lookback put option the predetermined maximum is $M=110$; for the UIC option the barrier and the strike price are given by $H=120$ and $K=100$, respectively. The expiration dates for both options are the same: $T=1$. The risk-free rate is $r=5 \%$. The parameters used in the double exponential jump diffusion are $\sigma=0.2$, $p=0.3,1 / \eta_{1}=0.02,1 / \eta_{2}=0.04, \lambda=3, S(0)=100$. To make a comparison with the limiting geometric Brownian motion model $(\lambda=0)$, we also use $\lambda=0.01$. The results are given in Table 3. All the computations are done on a Pentium 700 PC.

Monte Carlo simulation results are also reported in the table. Note that the Monte Carlo initial input). The main disadvantage is that it needs high accuracy computation, as it involves calculation of some factorial terms; typically 30-80 digit accuracy is needed. However, in many software packages (e.g. "Mathematica") one can specify arbitrary accuracy, and standard subroutines for high precision calculation in various programming languages (e.g. $\mathrm{C}++$ ) are readily available. 
simulation has two sources of errors: the random sampling error and systematic discretization bias. It is quite possible to significantly reduce the random sampling error here (thus the width of the confidence intervals) by using some variance reduction techniques, such as control variates and importance sampling. However, the systematic discretization bias, resulting from approximating the maximum of a continuous time process by the maximum of a discrete time process in simulation, is very difficult to be reduced. For both the lookback put and the UIC, it makes the calculation from the simulation biased low. Even in the Brownian motion case, because of the presence of boundary, this type of discretization bias is very significant, resulting in a surprisingly slow rate of convergence ${ }^{12}$ in simulating the first passage time, both theoretically and numerically. In the presence of jumps, the discretization bias could be even more serious, especially for large $T$ or large jump parameters.

\subsection{Perpetual American Options}

To simplify the derivation, we shall only focus on the perpetual American put option, as the methodology is valid for the perpetual American call option with dividends as well. Under the jump diffusion model, the price of an American put option is given by $\psi(S(0))=$ $\sup _{\tau} \mathrm{E}^{*}\left[e^{-r \tau}(K-S(\tau))^{+}\right]=\sup _{\tau} \mathrm{E}^{*}\left[e^{-r \tau}\left(K-S(0) e^{X(\tau)}\right)^{+}\right]$, where the supremum is taken over all stopping times $\tau$ taking values in $[0, \infty]$.

Theorem 4.3. Using $^{13}$ the notation $\beta_{3, r}$ and $\beta_{4, r}$ as in (4), the value ${ }^{14}$ of the perpetual American-put option is given by $\psi(S(0))=V(S(0))$, where the value function $V$ is given by

$$
V(v)=\left\{\begin{array}{cc}
K-v & ; \quad \text { if } v<v_{0} \\
A v^{-\beta_{3, r}}+B v^{-\beta_{4, r}} & ; \quad \text { if } v \geq v_{0}
\end{array},\right.
$$

\footnotetext{
${ }^{12}$ Asmussen, Glynn, and Pitman (1995) showed that theoretically the discretization error has an order $1 / 2$, which is much slower than the order 1 convergence for simulation without the boundary; 16,000 points are suggested in the paper for a Brownian motion with drift -1 and volatility $\sigma=1$ and time $T=8$.

${ }^{13}$ Actually, $\beta_{1, r}=1$.

${ }^{14}$ Gerber and Shiu (1998) and Mordecki (1999) study the same optimal stopping problem with one-sided jumps (can only jump up or down); this may not have the overshoot problem if the process always jumps away from (not jump towards) the boundary. Also $r=0$ in Mordecki (1999). Here we focus on the (two-sided) double exponential jump diffusion processes with $r \geq 0$.
} 
where

$$
\begin{gathered}
v_{0}=K \frac{\eta_{2}+1}{\eta_{2}} \cdot \frac{\beta_{3, r}}{1+\beta_{3, r}} \cdot \frac{\beta_{4, r}}{1+\beta_{4, r}}, \\
A=v_{0}^{\beta_{3, r}} \frac{1+\beta_{4, r}}{\beta_{4, r}-\beta_{3, r}}\left[\frac{\beta_{4, r}}{1+\beta_{4, r}} K-v_{0}\right]>0, \quad B=v_{0}^{\beta_{4, r}} \frac{1+\beta_{3, r}}{\beta_{4, r}-\beta_{3, r}}\left[v_{0}-\frac{\beta_{3, r}}{1+\beta_{3, r}} K\right]>0 .
\end{gathered}
$$

Furthermore, the optimal stopping time is given by $\tau^{*}=\inf \left\{t \geq 0: S(t) \leq v_{0}\right\}$.

The proof ${ }^{15}$ will be given in Appendix B.3. Note that the solution given in (12) satisfies the smooth-fit principle (i.e., the value function is continuous and continuously differentiable across the free boundary $\left.v_{0}\right)^{16}$.

Figure 2 graphs of the value of a perpetual American put option versus its parameters, $S(0), \eta_{1}, \eta_{2}, p, \lambda$. The defaulting parameters are $r=0.06, \sigma=0.20, K=100, S(0)=100$, $\lambda=3, p=0.3,1 / \eta_{1}=0.02$, and $1 / \eta_{2}=0.03$. It only takes less than one second to generate all the pictures in Figure 2 on a Pentium 700 PC. Not surprisingly, Figure 2 indicates that the option value is a decreasing function of $S(0), p$, and is an increasing function of $\lambda, 1 / \eta_{2}$, and $\sigma$, as it is a put option. What is interesting is that the option value is an increasing function of $1 / \eta_{1}$, which is the mean of the positive jumps. The reason is that the risk neutral drift also depends on $\eta_{1}$; a similar phenomenon was also pointed out in Merton (1976).

\section{Concluding Remarks}

Both the normal jump diffusion model and the double exponential jump diffusion model are special cases of the affine jump diffusion models (Duffie, Pan, and Singleton 2000, and Chacko and Das 2002), which include stochastic volatility and jumps in the volatility, and

\footnotetext{
${ }^{15}$ A result similar to (12) is also independently obtained by Mordecki (2002). However, there are two key differences. First, our proof not only covers the case of the perpetual American options, but also solves another infinite horizon free boundary problem (with a more complicated boundary condition) of (15) and (16), arising in approximating the finite horizon American options; see Appendix A.1. Secondly, the proof in Mordecki (2002) shows the results indirectly, as it first derives some general representations for Lévy processes, and then shows that the representations can be computed explicitly if the jump sizes are exponentially distributed. Here we prove and calculate the result directly by using martingale and PDE methods, without appealing to more general results from Lévy processes.

${ }^{16}$ The smooth-fit principle may not hold for general Lévy processes; see Pham (1997), Boyarchenkov and Levendorskii (2002a), where sufficient conditions for the smooth-fit principle are given.
} 


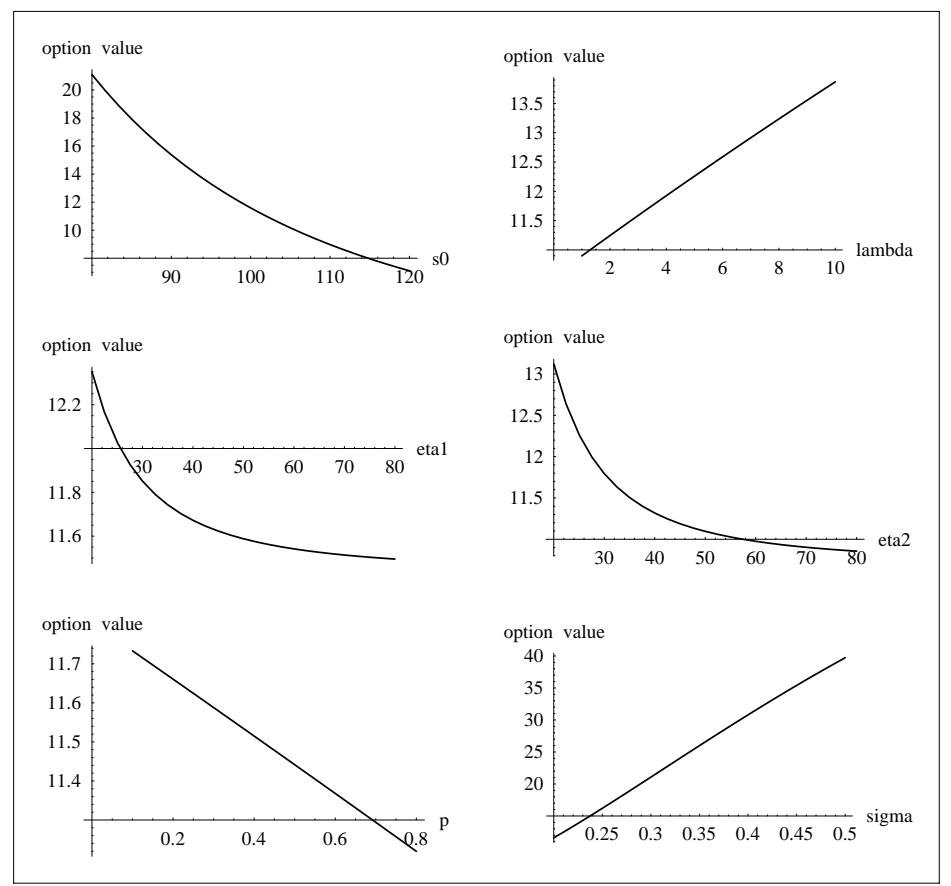

Figure 2: Values of American put options

of Lévy processes, which have independent increaments but with more general distributions. Whether the double exponential jump diffusion model is suitable for modeling purposes is ultimately a choice between analytical tractability and reality; and it should be judged on a case-by-case basis. (For example, the independent increament assumption is perhaps more defensible in the case of currency markets than in the case of equity markets.) See Cont and Tankov (2002) for calibration of the double exponential jump diffusion model to market data, and some empirical comparison with other models.

It is worthing mentioning that jump diffusion models may be also useful in modeling credit risks. In fact, Huang and Huang (2003) has used the double exponential jump diffusion model to study credit spread, and the empirical results there seem to be promising.

¿From a broader perspective, the paper calls for consideration of using exponential type distributions in modeling jumps in asset pricing, and, by understanding the simplest cases first, the results may hopefully shed some light on more general models with jumps.

Acknowledgments. We thank an anonymous referee, the associate editor and the editor 
for many helpful comments. We are also grateful to many people who offered insights into this work including seminar participants at various universities, and conference participants at Risk Annual Boston Meeting 2000, UCLA Mathematical Finance Conference 2001, Taipei International Quantitative Finance Conference 2001, Fifth SIAM Conference on Control and Its Applications 2001, American Finance Association Annual Meeting 2001, and the 11th Derivatives Conference in New York City 2003. We would like to thank Giovanni Petrella for his assistance in computing the numerical values of American options. All errors are ours alone.

\section{A Appendix: Derivation of the Approximation (7)}

\section{A.1 Outline of the Main Steps in the Derivation}

Let $t$ be the remaining time to maturity. Suppose the optimal exercise boundary is $v_{0}(t)$; in other words, it is optimal to exercise the option whenever the stock price falls below $v_{0}(t)$. Letting $x_{0}(t)=\log \left(v_{0}(t)\right), x=\log (v)$, and using the generator $\mathcal{L}$ in $(5)$, the value function $V(x, t)=\psi\left(e^{x}, t\right)$ and the optimal exercise boundary $v_{0}(t)$ must satisfy the free boundary problem: $-V_{t}-r V+\mathcal{L} V=0$, for $x>x_{0}(t)$; and $V(x, t)=K-e^{x}$, for $x \leq x_{0}(t)$. Define the early exercise premium as $\varepsilon(x, t):=V(x, t)-\operatorname{EuP}\left(e^{x}, t\right)$. Since the European put price satisfies the equation $-\mathrm{EuP}_{t}-r \mathrm{EuP}+\mathcal{L} \mathrm{EuP}=0$, for all $x$, it follows that the early exercise premium satisfies the equation

$$
-\varepsilon_{t}-r \varepsilon+\mathcal{L} \varepsilon=0, \quad \forall x>x_{0}(t) ; \quad \varepsilon(x, t)=K-e^{x}-\operatorname{EuP}\left(e^{x}, t\right), \forall x \leq x_{0}(t) .
$$

Introduce the change of variable $z=1-e^{-r t}, g(x, z)=\varepsilon(x, t) / z$. It is easy to see that $z_{t}=r e^{-r t}, \varepsilon_{x}=z g_{x}, \varepsilon_{x x}=z g_{x x}, \varepsilon_{t}=z_{t} g+z g_{z} z_{t}$. Plugging this back into (13), and dividing $z$ on both sides, we have

$$
-r(1-z) g_{z}-\left(\frac{r}{z}+\lambda\right) g+\frac{1}{2} \sigma^{2} g_{x x}+\left(r-\frac{1}{2} \sigma^{2}-\lambda \xi\right) g_{x}+\lambda \int_{-\infty}^{\infty} g(x+y, z) f_{Y}(y) d y=0
$$

for all $x>x_{0}(t)$ and $g(x, z)=\frac{1}{z}\left(K-e^{x}-\operatorname{EuP}\left(e^{x}, t\right)\right), \forall x \leq x_{0}(t)$. Following Barone-Adesi 
and Whaley (1987), the approximation will set

$$
(1-z) g_{z} \approx 0
$$

This is a reasonable assumption especially for very big or very small $t$. Indeed, as $t \rightarrow 0$, $1-z \rightarrow 0$, while as $t \rightarrow \infty, g_{z} \rightarrow 0$, because $g(x, z)$ converges to the price of a perpetual American put option. This also explains why in the numerical tables the error tends to be larger when $t=1$.

With the approximation (14), the function $g$ satisfies the following equations:

$$
-\left(\frac{r}{z}+\lambda\right) g+\frac{1}{2} \sigma^{2} g_{x x}+\left(r-\frac{1}{2} \sigma^{2}-\lambda \zeta\right) g_{x}+\lambda \int_{-\infty}^{\infty} g(x+y, z) f_{Y}(y) d y=0, \quad \forall x>x_{0}(t)
$$

and

$$
g(x, z)=\frac{1}{z}\left(K-e^{x}-\operatorname{EuP}\left(e^{x}, t\right)\right), \quad \forall x \leq x_{0}(t) .
$$

If we regard $t$, and hence $z$ and $x_{0}(t)$, to be fixed, the above equation becomes an ordinary integral-differential equation with free-boundary $x_{0}(t)$. Note that the boundary condition in (16) involves the European put option price $\operatorname{EuP}\left(e^{x}, t\right)$, which makes solving the free boundary

problem more difficult than that for perpetual American options. Under the assumption of exponential jump size distribution, however, the above free boundary problem can be solved explicitly as in Appendix A.2, resulting in the approximation in (7).

\section{A.2 Solving the Free Boundary Problem (15) and (16)}

Lemma A.1. Define

$$
\tilde{V}(x)=\left\{\begin{array}{cl}
K-e^{x}-h(x) & , \quad \text { if } x<x_{0} \\
A e^{-x \beta_{3}}+B e^{-x \beta_{4}} & , \quad \text { if } x \geq x_{0}
\end{array} ;\right.
$$

here $\beta_{3}, \beta_{4}>0, x_{0} \in(-\infty, \infty)$ are arbitrary constants, and $h(x)$ arbitrary continuous function. Then for any constant $b$, we have for all $x>x_{0}$,

$$
\begin{aligned}
& (-b \tilde{V}+\mathcal{L} \tilde{V})(x)=A e^{-x \beta_{3}} \tilde{f}\left(\beta_{3}\right)+B e^{-x \beta_{4}} \tilde{f}\left(\beta_{4}\right) \\
& \quad+\lambda q \eta_{2} e^{\left(x_{0}-x\right) \eta_{2}}\left[\frac{K}{\eta_{2}}-\frac{e^{x_{0}}}{1+\eta_{2}}-\frac{A e^{-x_{0} \beta_{3}}}{\eta_{2}-\beta_{3}}-\frac{B e^{-x_{0} \beta_{4}}}{\eta_{2}-\beta_{4}}-\int_{-\infty}^{0} h\left(x_{0}+y\right) e^{y \eta_{2}} d y\right]
\end{aligned}
$$


where $\tilde{f}(x):=G(-x)-b$.

Proof. First, we want to compute $\int_{-\infty}^{\infty} \tilde{V}(x+y) d F(y)$, which is essential to compute the generator $(\mathcal{L} \tilde{V})(x)$. For $x>x_{0}$, we have

$$
\begin{aligned}
& \int_{-\infty}^{\infty} \tilde{V}(x+y) d F(y) \\
= & \int_{-\infty}^{x_{0}-x}\left(K-e^{y+x}-h(x+y)\right) q \eta_{2} e^{y \eta_{2}} d y+\int_{x_{0}-x}^{0}\left(A e^{-\beta_{3}(y+x)}+B e^{-\beta_{4}(y+x)}\right) q \eta_{2} e^{y \eta_{2}} d y \\
& \quad+\int_{0}^{\infty}\left(A e^{-\beta_{3}(y+x)}+B e^{-\beta_{4}(y+x)}\right) p \eta_{1} e^{-y \eta_{1}} d y \\
= & q e^{\left(x_{0}-x\right) \eta_{2}}\left[K-\frac{\eta_{2} e^{x_{0}}}{1+\eta_{2}}\right]+\frac{q \eta_{2} A}{\eta_{2}-\beta_{3}}\left[e^{-\beta_{3} x}-e^{-\beta_{3} x_{0}} \cdot e^{\left(x_{0}-x\right) \eta_{2}}\right]-\int_{-\infty}^{x_{0}-x} h(x+y) q \eta_{2} e^{y \eta_{2}} d y \\
& +\frac{q \eta_{2} B}{\eta_{2}-\beta_{4}}\left[e^{-\beta_{4} x}-e^{-\beta_{4} x_{0}} \cdot e^{\left(x_{0}-x\right) \eta_{2}}\right]+\left[A \frac{p \eta_{1} e^{-\beta_{3} x}}{\eta_{1}+\beta_{3}}+B \frac{p \eta_{1} e^{-\beta_{4} x}}{\eta_{1}+\beta_{4}}\right] .
\end{aligned}
$$

Next, for $x>x_{0}$, we have

$$
\begin{aligned}
& (-b \tilde{V}+\mathcal{L} \tilde{V})(x) \\
& =\frac{1}{2} \sigma^{2}\left(A \beta_{3}^{2} e^{-x \beta_{3}}+B \beta_{4}^{2} e^{-x \beta_{4}}\right)+\left(r-\frac{1}{2} \sigma^{2}-\lambda \zeta\right)\left(-A \beta_{3} e^{-x \beta_{3}}-B \beta_{4} e^{-x \beta_{4}}\right) \\
& \quad-b\left(A e^{-x \beta_{3}}+B e^{-x \beta_{4}}\right)-\lambda\left(A e^{-x \beta_{3}}+B e^{-x \beta_{4}}\right)+\lambda\left\{q e^{\left(x_{0}-x\right) \eta_{2}}\left[K-\frac{\eta_{2} e^{x_{0}}}{1+\eta_{2}}\right]\right. \\
& \quad+\frac{q \eta_{2} A}{\eta_{2}-\beta_{3}}\left[e^{-\beta_{3} x}-e^{-\beta_{3} x_{0}} \cdot e^{\left(x_{0}-x\right) \eta_{2}}\right]-q \eta_{2} e^{\left(x_{0}-x\right) \eta_{2}} \int_{-\infty}^{0} h\left(x_{0}+y\right) e^{y \eta_{2}} d y \\
& \left.+\frac{q \eta_{2} B}{\eta_{2}-\beta_{4}}\left[e^{-\beta_{4} x}-e^{-\beta_{4} x_{0}} \cdot e^{\left(x_{0}-x\right) \eta_{2}}\right]+\left[A \frac{p \eta_{1} e^{-\beta_{3} x}}{\eta_{1}+\beta_{3}}+B \frac{p \eta_{1} e^{-\beta_{4} x}}{\eta_{1}+\beta_{4}}\right]\right\} \\
& =A e^{-x \beta_{3}} \tilde{f}\left(\beta_{3}\right)+B e^{-x \beta_{4}} \tilde{f}\left(\beta_{4}\right) \\
& +\lambda q e^{\left(x_{0}-x\right) \eta_{2}}\left[K-\frac{\eta_{2} e^{x_{0}}}{1+\eta_{2}}-\frac{\eta_{2} A e^{-x_{0} \beta_{3}}}{\eta_{2}-\beta_{3}}-\frac{\eta_{2} B e^{-x_{0} \beta_{4}}}{\eta_{2}-\beta_{4}}-\eta_{2} \int_{-\infty}^{0} h\left(x_{0}+y\right) e^{y \eta_{2}} d y\right],
\end{aligned}
$$

from which the proof is terminated.

Lemma A.2. For every $x_{0}$, we have

$$
\begin{aligned}
\left.\frac{\partial}{\partial x} \operatorname{EuP}\left(e^{x}, t\right)\right|_{x=x_{0}}= & \operatorname{EuP}\left(e^{x_{0}}, t\right)-K e^{-r t} \mathrm{P}^{*}\left(S(t) \leq K \mid S(0)=e^{x_{0}}\right), \\
\int_{-\infty}^{0} \operatorname{EuP}\left(e^{x_{0}+y}, t\right) e^{\eta_{2} y} d y= & \frac{1}{\eta_{2}+1} \operatorname{EuP}\left(e^{x_{0}}, t\right)+\frac{K e^{-r t}}{\eta_{2}\left(\eta_{2}+1\right)} \mathrm{P}^{*}\left(S(t) \leq K \mid S(0)=e^{x_{0}}\right) \\
& +\frac{K e^{-r t}}{\eta_{2}\left(\eta_{2}+1\right)} \cdot \mathrm{E}^{*}\left[(S(t) / K)^{-\eta_{2}} 1_{\{S(t)>K\}} \mid S(0)=e^{x_{0}}\right] .
\end{aligned}
$$


Proof. We have

$$
\begin{aligned}
\operatorname{EuP}\left(e^{x}, t\right) & =\mathrm{E}^{*}\left[e^{-r t}\left(K-e^{x} e^{X(t)}\right)^{+}\right]=\mathrm{E}^{*} \int_{-\infty}^{K-e^{x} e^{X(t)}} e^{-r t} 1_{\{y \geq 0\}} d y \\
& =\mathrm{E}^{*} \int_{e^{x}}^{\infty} e^{-r t} e^{X(t)} 1_{\left\{K-z e^{X(t)} \geq 0\right\}} d z=\int_{e^{x}}^{\infty} \mathrm{E}^{*}\left[e^{-r t} e^{X(t)} 1_{\left\{K-z e^{X(t)} \geq 0\right\}}\right] d z .
\end{aligned}
$$

Hence

$$
\left.\frac{\partial}{\partial x} \operatorname{EuP}\left(e^{x}, t\right)\right|_{x=x_{0}}=-e^{x_{0}} \cdot \mathrm{E}^{*}\left\{e^{-r t} e^{X(t)} 1_{\left\{K-e^{x_{0}} e^{X(t)} \geq 0\right\}}\right\},
$$

from which the first equation follows readily. As for the second equation, we have

$$
\begin{aligned}
& \int_{-\infty}^{0} \operatorname{EuP}\left(e^{x_{0}+y}, t\right) e^{\eta_{2} y} d y=\mathrm{E}^{*} \int_{-\infty}^{0} e^{-r t}\left(K-e^{x_{0}+y} e^{X(t)}\right)^{+} \cdot e^{\eta_{2} y} d y \\
& =e^{-r t} \mathrm{E}^{*} \int_{-\infty}^{0} 1_{\left\{K-e^{x_{0}} e^{X(t)} \geq 0\right\}} e^{\eta_{2} y}\left(K-e^{x_{0}+y} e^{X(t)}\right) d y \\
& \quad+e^{-r t} \mathrm{E}^{*} \int_{-\infty}^{\log \left(\frac{K}{e^{X(t)}}\right)-x_{0}} 1_{\left\{K-e^{x_{0}} e^{X(t)}<0\right\}} e^{\eta_{2} y}\left(K-e^{x_{0}+y} e^{X(t)}\right) d y \\
& =e^{-r t} \mathrm{E}^{*}\left[\left(\frac{K}{\eta_{2}}-\frac{S(t)}{\eta_{2}+1}\right) 1_{\{K-S(t) \geq 0\}}\right]+\frac{K^{\eta_{2}+1}}{\eta_{2}\left(\eta_{2}+1\right)} \cdot e^{-r t} \mathrm{E}^{*}\left[(S(t))^{-\eta_{2}} 1_{\{K-S(t)<0\}}\right],
\end{aligned}
$$

from which the conclusion follows.

Now we are in a position to solve the free boundary problem of (15) and (16). Since $\varepsilon(x, t)=z g(x, t)$, it is not difficult to see that (15)-(16) reduce to $-\frac{r}{z} \varepsilon+\mathcal{L} \varepsilon=0, \quad \forall x>x_{0}(t) ;$ $\varepsilon(x, t)=K-e^{x}-\operatorname{Eup}\left(e^{x}, t\right), \quad \forall x \leq x_{0}(t)$. Note we regard $t$ as fixed. Denote $x_{0}=x_{0}(t)$. By Lemma A.1, for $\varepsilon=A e^{-\beta_{3} x}+B e^{-\beta_{4} x}$ for $x \geq x_{0}$, we must have $G\left(-\beta_{3}\right)-\frac{r}{z}=0$, $G\left(-\beta_{4}\right)-\frac{r}{z}=0$, and

$$
\frac{K}{\eta_{2}}-\frac{e^{x_{0}}}{1+\eta_{2}}-\int_{-\infty}^{0} \operatorname{EuP}\left(e^{x_{0}+y}, t\right) \cdot e^{\eta_{2} y} d y=\frac{A e^{-\beta_{3} x_{0}}}{\eta_{2}-\beta_{3}}+\frac{B e^{-\beta_{4} x_{0}}}{\eta_{2}-\beta_{4}}
$$

The smooth-fit principle (i.e. the value function is continuous and continuously differentiable across the free boundary) yields two more equations $K-e^{x_{0}}-\operatorname{EuP}\left(e^{x_{0}}, t\right)=A e^{-\beta_{3} x_{0}}+B e^{-\beta_{4} x_{0}}$, $e^{x_{0}}+\left.\frac{\partial}{\partial x} \operatorname{EuP}\left(e^{x}, t\right)\right|_{x=x_{0}}=A \beta_{3} e^{-\beta_{3} x_{0}}+B \beta_{4} e^{-\beta_{4} x_{0}}$. These five equations determines the five unknown parameters $A, B, x_{0}, \beta_{3}$ and $\beta_{4}$. It is not difficult to verify that $A$ and $B$ are given 
by

$$
\begin{aligned}
& A=e^{\beta_{3} x_{0}} \cdot \frac{1}{\beta_{4}-\beta_{3}}\left[\beta_{4}\left(K-e^{x_{0}}-\operatorname{EuP}\left(e^{x_{0}}, t\right)\right)-\left(e^{x_{0}}+\left.\frac{\partial}{\partial x} \operatorname{EuP}\left(e^{x}, t\right)\right|_{x=x_{0}}\right)\right], \\
& B=e^{\beta_{4} x_{0}} \cdot \frac{1}{\beta_{3}-\beta_{4}}\left[\beta_{3}\left(K-e^{x_{0}}-\operatorname{EuP}\left(e^{x_{0}}, t\right)\right)-\left(e^{x_{0}}+\left.\frac{\partial}{\partial x} \operatorname{EuP}\left(e^{x}, t\right)\right|_{x=x_{0}}\right)\right] .
\end{aligned}
$$

After some algebra, (17) yields that the free boundary $x_{0}$ must satisfy the equation:

$$
\begin{aligned}
& \frac{\beta_{3} \beta_{4}}{\eta_{2}} K-e^{x_{0}} \frac{\beta_{3}+\beta_{4}+1+\beta_{4} \beta_{3}}{1+\eta_{2}} \\
= & \left.\frac{\partial}{\partial x} \operatorname{EuP}\left(e^{x}, t\right)\right|_{x=x_{0}}+\left(\beta_{3}+\beta_{4}-\eta_{2}\right) \operatorname{EuP}\left(e^{x_{0}}, t\right)+\left(\eta_{2}-\beta_{4}\right)\left(\eta_{2}-\beta_{3}\right) \int_{-\infty}^{0} \operatorname{EuP}\left(e^{x_{0}+y}, t\right) \cdot e^{\eta_{2} y} d y .
\end{aligned}
$$

Using Lemma A.2, we have

$$
\begin{aligned}
& A=\frac{e^{\beta_{3} x_{0}}}{\beta_{4}-\beta_{3}}\left\{\beta_{4} K-\left(1+\beta_{4}\right)\left[e^{x_{0}}+\operatorname{EuP}\left(e^{x_{0}}, t\right)\right]+K e^{-r t} \mathrm{P}^{*}\left[S(t) \leq K \mid S(0)=e^{x_{0}}\right]\right\}, \\
& B=\frac{e^{\beta_{4} x_{0}}}{\beta_{3}-\beta_{4}}\left\{\beta_{3} K-\left(1+\beta_{3}\right)\left[e^{x_{0}}+\operatorname{EuP}\left(e^{x_{0}}, t\right)\right]+K e^{-r t} \mathrm{P}^{*}\left[S(t) \leq K \mid S(0)=e^{x_{0}}\right]\right\},
\end{aligned}
$$

which are exactly (8) and (9). Again using Lemma A.2, we have that $x_{0}$ must satisfy

$$
\begin{aligned}
& \frac{\beta_{3} \beta_{4}}{\eta_{2}} K-e^{x_{0}} \frac{\left(1+\beta_{3}\right)\left(1+\beta_{4}\right)}{1+\eta_{2}} \\
= & \operatorname{EuP}\left(e^{x_{0}}, t\right) \frac{\left(1+\beta_{4}\right)\left(1+\beta_{3}\right)}{\eta_{2}+1}+\frac{\beta_{4} \beta_{3}-\eta_{2}-\eta_{2} \beta_{3}-\beta_{4} \eta_{2}}{\eta_{2}\left(\eta_{2}+1\right)} K e^{-r t} \mathrm{P}^{*}\left[S(t) \leq K \mid S(0)=e^{x_{0}}\right] \\
& +\left(\eta_{2}-\beta_{4}\right)\left(\eta_{2}-\beta_{3}\right) \cdot \frac{K e^{-r t}}{\eta_{2}\left(\eta_{2}+1\right)} \mathrm{E}^{*}\left[(S(t) / K)^{-\eta_{2}} I\{S(t) \geq K\} \mid S(0)=e^{x_{0}}\right] .
\end{aligned}
$$

Since $\eta_{2}$ is typically very large, as $1 / \eta_{2}$ is about $2 \%$ to $10 \%$, the last term in the above equation is generally very small, as the expectation $\mathrm{E}^{*}\left[(S(t) / K)^{-\eta_{2}} I\{S(t) \geq K\} \mid S(0)=e^{x_{0}}\right]$ is typically small. Ignoring the last term, we have that $x_{0}$ must satisfy the equation

$$
\begin{aligned}
\frac{\beta_{3} \beta_{4}}{\eta_{2}} K & -e^{x_{0}} \frac{\left(1+\beta_{3}\right)\left(1+\beta_{4}\right)}{1+\eta_{2}}=\operatorname{EuP}\left(e^{x_{0}}, t\right) \cdot \frac{\left(1+\beta_{4}\right)\left(1+\beta_{3}\right)}{\eta_{2}+1} \\
& +\frac{-\eta_{2}-\eta_{2} \beta_{3}-\beta_{4} \eta_{2}+\beta_{4} \beta_{3}}{\eta_{2}\left(\eta_{2}+1\right)} K e^{-r t} \cdot \mathrm{P}^{*}\left[S(t) \leq K \mid S(0)=v_{0}\right],
\end{aligned}
$$

which is exactly $(6)$.

It remains to show that $A>0$ and $B>0$. To do this, we need the following lemma. 
Lemma A.3. For the unique solution $v_{0}$ in equation (6), we have

$$
K>v_{0}+\operatorname{EuP}\left(v_{0}, t\right)=e^{-r t} \mathrm{E}^{*}\left[\max (S(t), K) \mid S(0)=v_{0}\right]
$$

Proof. We show this by contradiction. First, note that $v_{0}+\operatorname{EuP}\left(v_{0}, t\right)=e^{-r t} E^{v_{0}}[\max (S(t), K)]$ is an increasing function of $v_{0}$. Next, assume by contradiction that $K \leq v_{0}+\operatorname{EuP}\left(v_{0}, t\right)$. Since $C_{\beta}-D_{\beta}=\beta_{4} \beta_{3}-\eta_{2}\left(1+\beta_{3}+\beta_{4}\right)<0$, we have

$$
C_{\beta} K-D_{\beta}\left[v_{0}+\operatorname{EuP}\left(v_{0}, t\right)\right] \leq\left(C_{\beta}-D_{\beta}\right) K<\left(C_{\beta}-D_{\beta}\right) K e^{-r t} \cdot \mathrm{P}^{v_{0}}[S(t) \leq K]
$$

Thus, the left side of (6) would be smaller than the right side of (6); a contradiction.

Now we can show that $A, B>0$. By taking derivative and then using (18), it is easy to see that the function

$$
C_{\beta} K-D_{\beta}\left[v_{0}+\operatorname{EuP}\left(v_{0}, t\right)\right]-\left(C_{\beta}-D_{\beta}\right) K e^{-r t} \cdot \mathrm{P}^{v_{0}}[S(t) \leq K]
$$

is strictly increasing in $\beta_{3}$ and strictly decreasing in $\beta_{4}$. Replacing $\beta_{3}$ by $\eta_{2}$ in (19) and observing $\beta_{3}<\eta_{2}$ and (6), we have $\beta_{4} K-\left(1+\beta_{4}\right)\left[v_{0}+\operatorname{EuP}\left(v_{0}, t\right)\right]+K e^{-r t} \cdot \mathrm{P}^{v_{0}}[S(t) \leq K]>0$, yielding $A>0$. Similarly, since $\beta_{4}>\eta_{2}$, replacing $\beta_{4}$ by $\eta_{2}$ in (19) yields $B>0$.

\section{B Appendix: Proofs for Other Path-dependent Op- tions}

\section{B.1 Proof of Theorem 4.1 for Lookback Options}

Lemma B.1. We have $\lim _{y \rightarrow \infty} e^{y} \mathrm{P}^{*}\left[M_{X}(T) \geq y\right]=0, \forall T \geq 0$.

Proof. It is not difficult to see that the process $\left\{e^{\theta X(t)-G(\theta) t} ; t \geq 0\right\}$ is a martingale for any $\theta \in\left(-\eta_{2}, \eta_{1}\right)$. Fix an $\theta \in\left(1, \eta_{1}\right)$ such that $G(\theta)>0(\operatorname{such} \theta$ always exists since $G(1)=r \geq 0)$. It follows that $e^{y} \mathrm{P}^{*}\left[M_{X}(T) \geq y\right]=e^{(1-\theta) y} \cdot e^{\theta y} \mathrm{P}^{*}\left[M_{X}(T) \geq y\right]=e^{(1-\theta) y} \cdot e^{\theta y} \mathrm{P}^{*}\left[\tau_{y} \leq T\right]$, where $\tau_{y}$ is the first passage time of process $X$ over level $y$; however, the second term in the previous equation is dominated by $e^{\theta y} \mathrm{P}^{*}\left[\tau_{y} \leq T\right] \leq \mathrm{E}^{*}\left[e^{\theta X\left(\tau_{y} \wedge T\right)}\right] \leq e^{G(\theta) T} \mathrm{E}^{*}\left[e^{\theta X\left(\tau_{y} \wedge T\right)-G(\theta) \cdot\left(\tau_{y} \wedge T\right)}\right]=$ $e^{G(\theta) T}$, where the last equality follows from the optional sampling theorem. Since $\theta>1$, the result follows readily. 
Now we are ready to prove Theorem 4.1. Note that we only need to compute the Laplace transform of $L(s, M ; T):=\mathrm{E}^{*}\left[e^{-r T} \max \left(M, s e^{M_{X}(T)}\right)\right], M \geq s$, where $s=S(0)$ and $M$ are constants, and $M_{X}(T):=\max _{0 \leq t \leq T} X(t)$. Letting $z=\log (M / s) \geq 0$, we have

$$
L(s, M ; T)=s \mathrm{E}^{*}\left[e^{-r T} \max \left(e^{z}, e^{M_{X}(T)}\right)\right]=s \mathrm{E}^{*}\left[e^{-r T}\left(e^{M_{X}(T)}-e^{z}\right) \mathbf{1}_{\left\{M_{X}(T) \geq z\right\}}\right]+s e^{z} e^{-r T} .
$$

Integration by parts yields

$$
\begin{gathered}
\mathrm{E}^{*}\left[e^{-r T} e^{M_{X}(T)} \mathbf{1}_{\left\{M_{X}(T) \geq z\right\}}\right]=-e^{-r T} \int_{z}^{\infty} e^{y} d \mathrm{P}^{*}\left[M_{X}(T) \geq y\right] \\
=-e^{-r T}\left\{-e^{z} \mathrm{P}^{*}\left[M_{X}(T) \geq z\right]-\int_{z}^{\infty} \mathrm{P}^{*}\left[M_{X}(T) \geq y\right] e^{y} d y\right\} \\
=\mathrm{E}^{*}\left[e^{-r T} e^{z} \mathbf{1}_{\left\{M_{X}(T) \geq z\right\}}\right]+e^{-r T} \int_{z}^{\infty} e^{y} \mathrm{P}^{*}\left[M_{X}(T) \geq y\right] d y
\end{gathered}
$$

here we have used Lemma B.1. It follows that $L(s, M ; T)=s e^{-r T} \int_{z}^{\infty} e^{y} \mathrm{P}^{*}\left[M_{X}(T) \geq y\right] d y+$ $M e^{-r T}$. Therefore, for any $\alpha>0$,

$$
\begin{aligned}
\int_{0}^{\infty} e^{-\alpha T} L(s, M ; T) d T & =s \int_{0}^{\infty} e^{-\alpha T} e^{-r T} \int_{z}^{\infty} e^{y} \mathrm{P}^{*}\left[M_{X}(T) \geq y\right] d y d T+\frac{M}{\alpha+r} \\
& =s \int_{z}^{\infty} e^{y} \int_{0}^{\infty} e^{-(\alpha+r) T} \mathrm{P}^{*}\left[M_{X}(T) \geq y\right] d T d y+\frac{M}{\alpha+r}
\end{aligned}
$$

However, it follows from Kou and Wang (2003) that

$$
\begin{gathered}
\int_{0}^{\infty} e^{-(\alpha+r) T} \mathrm{P}^{*}\left[M_{X}(T) \geq y\right] d T=A_{1} e^{-y \beta_{1, \alpha+r}}+B_{1} e^{-y \beta_{2, \alpha+r}} \\
A_{1}=\frac{1}{\alpha+r} \frac{\eta_{1}-\beta_{1, \alpha+r}}{\eta_{1}} \cdot \frac{\beta_{2, \alpha+r}}{\beta_{2, \alpha+r}-\beta_{1, \alpha+r}}, \quad B_{1}=\frac{1}{\alpha+r} \frac{\beta_{2, \alpha+r}-\eta_{1}}{\eta_{1}} \cdot \frac{\beta_{1, \alpha+r}}{\beta_{2, \alpha+r}-\beta_{1, \alpha+r}} .
\end{gathered}
$$

Note that $\beta_{2, \alpha+r}>\eta_{1}>1, \beta_{1, \alpha+r}>\beta_{1, r}=1$. Therefore,

$$
\begin{aligned}
\int_{0}^{\infty} e^{-\alpha T} L(s, M ; T) d T & =s \int_{z}^{\infty} e^{y} A_{1} e^{-y \beta_{1, \alpha+r}} d y+s \int_{z}^{\infty} e^{y} B_{1} e^{-y \beta_{2, \alpha+r}} d y+\frac{M}{\alpha+r} \\
& =s A_{1} \frac{e^{-z\left(\beta_{1, \alpha+r}-1\right)}}{\beta_{1, \alpha+r}-1}+s B_{1} \frac{e^{-z\left(\beta_{2, \alpha+r}-1\right)}}{\beta_{2, \alpha+r}-1}+\frac{M}{\alpha+r} \\
& =s \frac{A_{\alpha}}{C_{\alpha}} e^{-z\left(\beta_{1, \alpha+r}-1\right)}+s \frac{B_{\alpha}}{C_{\alpha}} e^{-z\left(\beta_{2, \alpha+r}-1\right)}+\frac{M}{\alpha+r} .
\end{aligned}
$$

This yields the Laplace transform we obtained in Theorem 4.1. 


\section{B.2 Proof of Theorem 4.2 for Barrier Options}

We can write UIC as

$$
\begin{aligned}
\mathrm{UIC} & =\mathrm{E}^{*}\left[e^{-r T}(S(T)-K)^{+} \mathbf{1}_{\left\{\max _{0 \leq t \leq T} S(t) \geq H\right\}}\right] \\
& =\mathrm{E}^{*}\left[e^{-r T} S(T) \mathbf{1}_{\left\{S(T) \geq K, \max _{0 \leq t \leq T} S(t) \geq H\right\}}\right]-K e^{-r T} \mathrm{P}^{*}\left[S(T) \geq K, \max _{0 \leq t \leq T} S(t) \geq H\right] \\
& =I-K e^{-r T} \cdot I I \text { (say). }
\end{aligned}
$$

It is easy to compute the second term, as

$$
I I=\Psi\left(r-\frac{1}{2} \sigma^{2}-\lambda \zeta, \sigma, \lambda, p, \eta_{1}, \eta_{2} ; \log (K / S(0)), \log (H / S(0)), T\right) .
$$

For the first term, we can use a change of numeraire argument. More precisely, introduce a new probability $\tilde{P}$ defined as

$$
\left.\frac{d \tilde{\mathrm{P}}}{d \mathrm{P}^{*}}\right|_{t=T}=e^{-r T} \frac{S(T)}{S(0)}=e^{-r T} e^{X(T)}=\exp \left\{\left(-\frac{1}{2} \sigma^{2}-\lambda \zeta\right) T+\sigma W(T)+\sum_{i=1}^{N(T)} Y_{i}\right\} .
$$

Note that this is a well defined probability as $\mathrm{E}^{*}\left\{e^{-r t} \frac{S(t)}{S(0)}\right\}=1$. We have, by the Girsanov theorem for jump processes, $\tilde{W}(t):=W(t)-\sigma t$ is a new Brownian motion under $\tilde{\mathrm{P}}$, and the original process

$$
X(t)=\left(r-\frac{1}{2} \sigma^{2}-\lambda \zeta\right) t+\sigma W(t)+\sum_{i=1}^{N(t)} Y_{i}=\left(r+\frac{1}{2} \sigma^{2}-\lambda \zeta\right) t+\sigma \tilde{W}(t)+\sum_{i=1}^{N(t)} Y_{i}
$$

is a new double exponential jump diffusion process with the Poisson process $N(t)$ having a new rate $\tilde{\lambda}=\lambda \mathrm{E}^{*}\left(e^{Y}\right)=\lambda(1+\zeta)$. and the jump sizes $Y$ 's are i.i.d. with a new density given by

$$
\begin{aligned}
& \frac{1}{\mathrm{E}^{*}\left(e^{Y}\right)} e^{y} f_{Y}(y)=\frac{1}{\mathrm{E}^{*}\left(e^{Y}\right)} e^{y} p \eta_{1} e^{-\eta_{1} y} \mathbf{1}_{\{y \geq 0\}}+\frac{1}{\mathrm{E}^{*}\left(e^{Y}\right)} e^{y} q \eta_{2} e^{\eta_{2} y} \mathbf{1}_{\{y<0\}} \\
& =p \frac{1}{\mathrm{E}^{*}\left(e^{Y}\right)} \cdot \frac{\eta_{1}}{\eta_{1}-1}\left(\eta_{1}-1\right) e^{-\left(\eta_{1}-1\right) y} \mathbf{1}_{\{y \geq 0\}}+q \frac{1}{\mathrm{E}^{*}\left(e^{Y}\right)} \cdot \frac{\eta_{2}}{\eta_{2}+1}\left(\eta_{2}+1\right) e^{\left(\eta_{2}+1\right) y} \mathbf{1}_{\{y<0\}} .
\end{aligned}
$$

Thus, it is still a double exponential density with $\tilde{\eta}_{1}=\eta_{1}-1, \tilde{\eta}_{2}=\eta_{2}+1$,

$$
\tilde{p}=p\left\{\frac{p \eta_{1}}{\eta_{1}-1}+\frac{q \eta_{2}}{\eta_{2}+1}\right\}^{-1} \frac{\eta_{1}}{\eta_{1}-1}, \quad \tilde{q}=q\left\{\frac{p \eta_{1}}{\eta_{1}-1}+\frac{q \eta_{2}}{\eta_{2}+1}\right\}^{-1} \frac{\eta_{2}}{\eta_{2}+1} .
$$


In summary, we have

$$
\begin{aligned}
I & =S(0) \mathrm{E}^{*}\left[e^{-r T} \frac{S(T)}{S(0)} \cdot \mathbf{1}_{\left\{S(T) \geq K, \max _{0 \leq t \leq T} S(t) \geq H\right\}}\right] \\
& =S(0) \tilde{\mathrm{P}}\left[S(T) \geq K, \min _{0 \leq t \leq T} S(t) \leq H\right] \\
& =S(0) \Psi\left(r+\frac{1}{2} \sigma^{2}-\lambda \zeta, \sigma, \tilde{\lambda}, \tilde{p}, \tilde{\eta}_{1}, \tilde{\eta}_{2} ; \log (K / S(0)), \log (H / S(0)), T\right)
\end{aligned}
$$

and UIC $=I-K e^{-r T} \cdot I I$, from which the conclusion follows.

\section{B.3 Proof of Theorem 4.3 for Perpetual American Options}

Lemma B.2. Suppose there exist some $x_{0}<\log K$ and a non-negative $\mathcal{C}^{1}$ function $u(x)$ such that: (1) the function $u$ is $C^{2}$ on $\mathcal{R} \backslash\left\{x_{0}\right\}$, and is convex with $u^{\prime \prime}\left(x_{0}-\right)$ and $u^{\prime \prime}\left(x_{0}+\right)$ existing; (2) $(\mathcal{L} u)(x)-r u(x)=0$ for all $x>x_{0}$; (3) $(\mathcal{L} u)(x)-r u(x)<0$ for all $x<x_{0}$; (4) $u(x)>\left(K-e^{x}\right)^{+}$for all $x>x_{0} ;(5) u(x)=\left(K-e^{x}\right)^{+}$for all $x \leq x_{0}$; (6) there exists a random variable $Z$ with $\mathrm{E}^{*}[Z]<\infty$, such that $e^{-r\left(t \wedge \tau \wedge \tau^{*}\right)} u\left(X\left(t \wedge \tau \wedge \tau^{*}\right)+x\right) \leq Z$, for any $t \geq 0, x$ and any stopping time $\tau$. Here the infinitesimal generator $\mathcal{L}$ is defined in (5). Then the option price $\psi(S(0))=u(\log (S(0)))$ and the optimal stopping time is given by $\tau^{*}:=\inf \left\{t \geq 0: \quad S(t) \leq e^{x_{0}}\right\}$.

Proof: Define $\tilde{X}(t)=x+X(t)$. Then $\tilde{X}(t)$ has the same generator $\mathcal{L}$. The result now follows from a similar argument in Mordecki (1999, p. 230-232), except with $M(t)$ being changed to $M(t):=e^{-r t} u(\tilde{X}(t))-\int_{0}^{t}\{-r u(\tilde{X}(s))+\mathcal{L} u(\tilde{X}(s))\} d s$.

Proof of Theorem 4.3. Let $x=\log (v)$ and $x_{0}=\log \left(v_{0}\right)$. Then

$$
V(x)=\left\{\begin{array}{cc}
K-e^{x} & ; \quad \text { if } x<x_{0} \\
A e^{-x \beta_{3, r}}+B e^{-x \beta_{4, r}} & ; \quad \text { if } x \geq x_{0}
\end{array}\right.
$$

For notation simplicity, we shall write $\beta_{3} \equiv \beta_{3, r}$ and $\beta_{4} \equiv \beta_{4, r}$. To prove Theorem 1 , we only need to check the conditions in Lemma A.1. Note that $f\left(\beta_{3}\right)=f\left(\beta_{4}\right)=0$, and

$$
\begin{aligned}
K-e^{x_{0}} & =A e^{-x_{0} \beta_{3}}+B e^{-x_{0} \beta_{4}}, \quad e^{x_{0}}=A \beta_{3} e^{-x_{0} \beta_{3}}+B \beta_{4} e^{-x_{0} \beta_{4}} \\
0 & =K-\frac{e^{x_{0}} \eta_{2}}{1+\eta_{2}}-\frac{A \eta_{2} e^{-x_{0} \beta_{3}}}{\eta_{2}-\beta_{3}}-\frac{B \eta_{2} e^{-x_{0} \beta_{4}}}{\eta_{2}-\beta_{4}}
\end{aligned}
$$


Therefore, Condition 2 follows from Lemma A.1 with $h=0$. Conditions 1, 4, 5, and 6 are trivial by noting that $V\left(x_{0}+\right)=V\left(x_{0}-\right)$ and $0 \leq V(x) \leq K$.

As to Condition 3 , note that for $x<x_{0}$, we have

$$
\begin{gathered}
\int_{-\infty}^{\infty} V(x+y) a d F(y)=\int_{-\infty}^{0}\left(K-e^{y+x}\right) q \eta_{2} e^{y \eta_{2}} d y+\int_{0}^{x_{0}-x}\left(K-e^{y+x}\right) p \eta_{1} e^{-y \eta_{1}} d y \\
+\int_{x_{0}-x}^{\infty}\left(A e^{-\beta_{3}(y+x)}+B e^{-\beta_{4}(y+x)}\right) p \eta_{1} e^{-y \eta_{1}} d y \\
=K-e^{x}\left[\frac{q \eta_{2}}{\eta_{2}+1}+\frac{p \eta_{1}}{\eta_{1}-1}\right]-p e^{-\eta_{1}\left(x_{0}-x\right)}\left[K-\frac{\eta_{1} e^{x_{0}}}{\eta_{1}-1}-A \frac{\eta_{1} e^{-x_{0} \beta_{3}}}{\eta_{1}+\beta_{3}}-B \frac{\eta_{1} e^{-x_{0} \beta_{4}}}{\eta_{1}+\beta_{4}}\right] .
\end{gathered}
$$

Therefore, for $x<x_{0}$,

$$
\begin{aligned}
& (-r V+\mathcal{L} V)(x)=-\frac{1}{2} \sigma^{2} e^{x}+\left(r-\frac{1}{2} \sigma^{2}-\lambda \zeta\right)\left(-e^{x}\right)-r\left(K-e^{x}\right)-\lambda\left(K-e^{x}\right) \\
& \quad+\lambda\left\{K-e^{x}\left[\frac{q \eta_{2}}{\eta_{2}+1}+\frac{p \eta_{1}}{\eta_{1}-1}\right]-p e^{-\eta_{1}\left(x_{0}-x\right)}\left[K-\frac{\eta_{1} e^{x_{0}}}{\eta_{1}-1}-A \frac{\eta_{1} e^{-\beta_{3} x_{0}}}{\eta_{1}+\beta_{3}}-B \frac{\eta_{1} e^{-\beta_{4} x_{0}}}{\eta_{1}+\beta_{4}}\right]\right\} .
\end{aligned}
$$

Rearranging terms and using (3) we have for $x<x_{0}$,

$$
(-r V+\mathcal{L} V)(x)=-r K-\lambda e^{-\eta_{1}\left(x_{0}-x\right)} p\left[K-\frac{\eta_{1} e^{x_{0}}}{\eta_{1}-1}-A \frac{\eta_{1} e^{-\beta_{3} x_{0}}}{\eta_{1}+\beta_{3}}-B \frac{\eta_{1} e^{-\beta_{4} x_{0}}}{\eta_{1}+\beta_{4}}\right]
$$

The right hand side can be further simplified as

$$
\begin{aligned}
& K-\frac{\eta_{1} e^{x_{0}}}{\eta_{1}-1}-\frac{\eta_{1} A e^{-\beta_{3} x_{0}}}{\eta_{1}+\beta_{3}}-\frac{\eta_{1} B e^{-\beta_{4} x_{0}}}{\eta_{1}+\beta_{4}} \\
= & K-\frac{\eta_{1} v_{0}}{\eta_{1}-1}-\frac{\eta_{1}}{\eta_{1}+\beta_{3}} \frac{1+\beta_{4}}{\beta_{4}-\beta_{3}}\left[\frac{\beta_{4}}{1+\beta_{4}} K-v_{0}\right]-\frac{\eta_{1}}{\eta_{1}+\beta_{4}} \frac{1+\beta_{3}}{\beta_{4}-\beta_{3}}\left[v_{0}-\frac{\beta_{3}}{1+\beta_{3}} K\right] \\
= & K \frac{\beta_{3} \beta_{4}}{\left(\eta_{1}+\beta_{3}\right)\left(\eta_{1}+\beta_{4}\right)}-v_{0} \eta_{1} \frac{\left(1+\beta_{3}\right)\left(1+\beta_{4}\right)}{\left(\eta_{1}-1\right)\left(\eta_{1}+\beta_{3}\right)\left(\eta_{1}+\beta_{4}\right)} \\
= & -K \frac{\beta_{3} \beta_{4}}{\left(\eta_{1}+\beta_{3}\right)\left(\eta_{1}+\beta_{4}\right)} \frac{\eta_{2}+\eta_{1}}{\eta_{2}\left(\eta_{1}-1\right)} .
\end{aligned}
$$

In summary we have for $x<x_{0}$,

$$
(-r V+\mathcal{L} V)(x)=-r K+p \lambda e^{-\eta_{1}\left(x_{0}-x\right)} K \frac{\beta_{3} \beta_{4}\left(\eta_{2}+\eta_{1}\right)}{\left(\eta_{1}+\beta_{3}\right)\left(\eta_{1}+\beta_{4}\right) \eta_{2}\left(\eta_{1}-1\right)},
$$

from which it is easy to see that $(-r V+\mathcal{L} V)(x)$ is an increasing function, thanks to the assumption $\eta_{1}>1$. Thus, to show Condition 3 it suffices to show that $(-r V+\mathcal{L} V)\left(x_{0}-\right) \leq 0$. 
However, since $V(x)$ is bounded and continuous, it follows from the Dominated Convergence Theorem that

$(\mathcal{L} V)\left(x_{0}-\right)-(\mathcal{L} V)\left(x_{0}+\right)=\frac{1}{2}\left(V^{\prime \prime}\left(x_{0}-\right)-V^{\prime \prime}\left(x_{0}+\right)\right)=-\frac{1}{2}\left(e^{x_{0}}+\beta_{3}^{2} A e^{-\beta_{3} x_{0}}+\beta_{4}^{2} B e^{-\beta_{2} x_{0}}\right) \leq 0$.

But $(-r V+\mathcal{L} V)\left(x_{0}+\right)=0$, which completes the proof.

\section{References}

[1] Amin, K. (1993). Jump diffusion option valuation in discrete time. J. Finance 48 18331863.

[2] Asmussen, S., P. Glynn, and J. Pitman. 1995. Discretization error in simulation of one-dimensional Brownian motion. Ann. Appl. Probab. 5 875-896.

[3] Avram, F., T. Chan, and M. Usabel. 2001. Pricing American options under spectrally negative exponential Lévy models. Working paper, Heriot-Watt University, Edinburgh.

[4] Barone-Adesi, G., R. E. Whaley. 1987. Efficient analytic approximations of American option values. J. of Finance $\mathbf{4 2}$ 301-320.

[5] Black, F., J. Cox. 1976. Valuing corporate debt: some effects of bond indenture provisions. J. Finance 31 351-367.

[6] Boyle, Ph., M. Broadie, P. Glasserman. 1997. Simulation methods for security pricing. J. Econom. Dynam. Control 21 1267-1321.

[7] Boyarchenko, S., S. Levendorskii. 2002a. Perpetual American options under Lévy processes. SIAM J. Control and Optimization 40 1663-1696.

[8] Boyarchenko, S., S. Levendorskii. 2002b. Barrier options and touch-and-out options under regular Lévy processes of exponential type. Ann. of Appl. Probab. 12 1261-1298.

[9] Broadie, M., J. Detemple. 1996. American option valuation: new bounds and approximations. Rev. Financial Stud. 9 1211-1250. 
[10] Broadie, M., P. Glasserman. 1997. Pricing American-style securities using simulation. J. Econom. Dynam. Control 21 1323-1352.

[11] Carr, P. 1998. Randomization and the American put. Rev. Financial Stud. 11 597-626.

[12] Carriére, J. 1996. Valuation of early-exercise price of options using simulations and nonparametric regressions. Insurance: Math. and Econom. 19 19-30.

[13] Chacko, G., S. R. Das. 2002. Pricing interest rate derivatives: a general approach. Rev. of Financial Stud., 15 195-241.

[14] Conze, A., R. Viswanathan. 1991. Path dependent options: the case of lookback options. J. Finance 46 1893-1907.

[15] Cont, R., P. Tankov. 2002. Calibration of jump-diffusion option pricing models: a robust non-parametric approach. Working paper, Ecole Polytechnique, Paris.

[16] Das, S. R., S. Foresi. 1996. Exact solutions for bond and option prices with systematic jump risk. Rev. of Derivatives Research 1 7-24.

[17] Davydov, D., V. Linetsky. 2001. The valuation and hedging of path-dependent options under the CEV process. Management Sci. 47 949-965.

[18] Davydov, D., V. Linetsky. 2003. Pricing options on scalar diffusions: an eigenfunction expansion approach. To appear in Operations Research.

[19] Duffie, D., J. Pan, K. Singleton. 2000. Transform analysis and option pricing for affine jump-diffusions. Econometrica. 68 1343-1376.

[20] Gerber, H. U., E. Shiu. 1998. Pricing perpetual options for jump processes. North American Acturial Journal 2 101-112.

[21] Geske, R., E. Johnson. 1984. The American put option valued analytically. J. Finance 39 1511-1524. 
[22] Goldman, M.B., H. B. Sosin, M. A. Gatto. 1979. Path-Dependent Options: Buy at the Low, Sell at the High. J. Finance 34 1111-1127.

[23] d'Halluin, J., P. Forsyth, K. Vetzal. 2003. Rubust numerical methods for contingent claims under jump diffusion processes. Working paper, Univ. of Waterloo.

[24] Haugh, M., L. Kogan. 2002. Pricing American Options: A Duality Approach. To appear in Operations Research.

[25] Huang, J., M. Huang. 2003. How much of the corporate-treasury yield spread is due to credit risk? Working paper, New York University and Stanford University.

[26] Hull, J. 2000. Options, Futures, and Other Derivative Securities. 4th edition, Prentice Hall, New Jersey.

[27] Ju, N. 1998. Pricing by American option by approximating its early exercise boundary as a multipiece exponential function. Rev. Financial Stud. 11 627-646.

[28] Kou, S. G. 2002. A jump diffusion model for option pricing. Management Sci. 48 10861101.

[29] Kou, S. G., H. Wang. 2003. First passage times for a jump diffusion process. Adv. Applied Probab. 35 504-531.

[30] Kyprianou, A., M. Pistorius. 2003. Perpetual options and canadization through fluctuation theory. To appear in Ann. Applied Prob.

[31] Leland, H. E. 1994. Corporate Debt Value, Bond Covenants, and Optimal Capital Structure. J. Finance 49 1213-1252.

[32] Longstaff, F. A. 1995. How Much Can Marketability Affect Security Values? J. Finance $501767-1774$.

[33] Longstaff, F. A., E. S. Schwartz. 1995. A Simple Approach to Valuing Risky Fixed and Floating Rate Debt. J. Finance 50 789-819. 
[34] Longstaff, F. A., E. S. Schwartz. 2001. Valuing American options by simulation: a simple least-squares approach. Rev. Financial Stud. 14 113-147.

[35] Lucas, R. E. 1978. Asset prices in an exchange economy. Econometrica 46 1429-1445.

[36] McDonald, R., D. Siegel. 1986. The value of waiting to invest. Quarterly J. of Economics $101707-727$.

[37] McKean, H. P. 1965. A free boundary problem for the heat equation arising from a problem in mathematical economics. Industrial Management Review 6 33-39.

[38] McMillan, L. 1986. Analytic approximation for the American put option. Advances in Futures and Options Research. 1 119-140.

[39] Merton, R. C. 1973. The theory of rational option pricing. Bell J. of Econom. and Management Sci. 4 141-183.

[40] Merton, R. C. 1974. On the pricing of corporate debt: the risk structure of interest rates. J. Finance 29 449-469.

[41] Merton, R. C. 1976. Option pricing when underlying stock returns are discontinuous. J. Financial Econom. 3 125-144.

[42] Mordecki, E. 1999. Optimal stopping for a diffusion with jumps. Finance and Stochastics $3227-236$.

[43] Mordecki, E. 2002. Optimal stopping and perpetual options for Lévy processes. Finance and Stochastics 6 273-293.

[44] Naik, V., M. Lee. 1990. General equilibrium pricing of options on the market portfolio with discontinuous returns. Rev. Financial Stud. 3 493-521.

[45] Petrella, G., S. G. Kou, H. Wang. 2003. Some Laplace transforms in option pricing. Working paper, Columbia University and Brown University. 
[46] Pham, H. 1997. Optimal stopping, free boundary and American option in a jumpdiffusion model. Appl. Math. and Optimization 35 145-164.

[47] Rogers, L. C. G. 2000. Evaluating first-passage probabilites for spectrally one-sided Lévy processes. J. Appl. Probab. 37 1173-1180.

[48] Rogers, L. C. G. 2002. Monte Carlo valuation of American options. Math. Finance 12 271-286.

[49] Schroder, M. 1999. Change of numeraire for pricing futures, forwards, and options. Rev. Financial Stud. 12 1143-1163.

[50] Siegmund, D. 1985. Sequential Analysis. Springer, New York.

[51] Sullivan, M. A. 2000. Valuing American put options using Gaussian quadrature. Rev. Financial Stud. 13 75-94.

[52] Tilley, J. A. 1993. Valuing American options in a path simulation model. Transactions of the Society of Acturaries 45 83-104.

[53] Tsitsiklis, J., B. van Roy. 1999. Optimal stopping of Markov processes: Hilbert space theory, approximation of algorithms, and an application to pricing high-dimensional financial derivatives. IEEE Transactions on Automatic Control 44 1840-1851.

[54] Zhang, X. L. 1997. Numerical analysis of American option pricing in a jump diffusion model. Math. of Operations Research 22 668-690. 